The Egyptian Journal of Hospital Medicine (April 2013) Vol. 51, Page 405- 421

\title{
The Role of Bee Venom in Controlling Albino Rats Fetal Morphometric and Morphological Changes by Carbimazole Induced Hypothyroidism
}

\author{
Abu Gabal $H^{*}$, Moamena $\mathbf{M}^{* *}$ and Al Moalla $\mathbf{H}^{* *}$ \\ * College of Science and Humanity Studies, Salman Bin Abdul Aziz University. \\ **College of Science, Princess Nora Bent Abdul Rahman University.
}

Aim of the work. The present study was planned to investigate the role played by Bee venom in improving the morphometric and morphological changes induced by carbimazole in pregnant albino rats and their embryos.

Materials and method .A total number of 60 mature virgin female and 30 male Wistar wistar albino rats (for fertilization) were used in this study.Pregnancy was ascertained by vaginal smears. The experimental animals were divided into the following groups:

I- (Normal group): in this group the pregnant rats were injected intraperitonealy by distilled water (The solvent of both carbimazole and bee venom) by dose $1 \mathrm{ml} / 200 \mathrm{~g}$.body weight, from day 1 to day 18 of gestation.

II-The group of treated animals: this group was divided into 3 subgroups:

1-The carbimazole group: this group was divided into2 subgroups, the pregnant rats were orally injected at a dose 2 and $3 \mathrm{mg} / 200 \mathrm{~g}$.body weight, daily from day 1 to day 18 of gestation.

2-The Bee venom group: the pregnant rats were intraperitonealy injected with a dose 0.6 $\mathrm{mg} / 200 \mathrm{~g}$. body weight on days $2,3,4,7,8,9,10,11,12,15$ and 16 of gestation.

3-The treatment group: the pregnant rats were intraperitonealy injected with Bee venom

at a dose $0.6 \mathrm{mg} / 200 \mathrm{~g}$. body weight 1 hour after the intraperitonealy injection by the 2 doses of carbimazole ( 2 and $3 \mathrm{mg} / 200 \mathrm{~g}$. body weight). The days of injection for both treatments (Bee venom and carbimazole) were as mentioned before.

Results. The results of the present study showed that carbimazole treatment with its 2 doses induced highly significant increase in the body weights of pregnant rats, highly significant reduction of the uterus weight with shortness of the horns as well as unequal distribution of the embryos between them, increased number of the resorbed fetuses when comparing with the control group, Bee venom injection revealed improvement of these changes .The orally injection of the 2 doses of carbimazole resulted in very highly decrease in fetuses body weights and lengths; however Bee venom induced obvious improvement as compared with the carbimazole effects. Orally injection of carbimazole at the dose $3 \mathrm{mg} / 200 \mathrm{~g}$ body weight showed increased fetal mortality rate as compared with the control group; however, intraperitonealy injection of Bee venom resulted in improvement in the rate of live fetuses and never of dead ones was observed after Bee venom treatment. The 2 doses of carbimazole induced lots of malformations of embryos such as variations in the size of embryos of the same mother, the embryos exhibited fragile skin, sub dermal blood coagulation beside edema in different regions of the fetal body, as well as malformations of the regions of the eye resembled in exophthalmos and rostrum region such as cleft lips, beside clubbed fore and hind limbs, kyphosis of the body of the embryos, exencephaly.Bee venom treatment resulted in control of the changes induced by carbimazole injection. The fetuses were resembled to those of the control group.

Conclusion: It is clear that bee venom plays an important role in controlling the morphometric and morphological changes in fetuses of albino rats suffering from hypothyroidism induced by carbimazole •

Key words: Carbimazole, Thyroid gland, Bee venom. Pregnant Rats, fetuses. 
The Role of Bee Venom in Controlling the Morphometric and Morphological Changes...

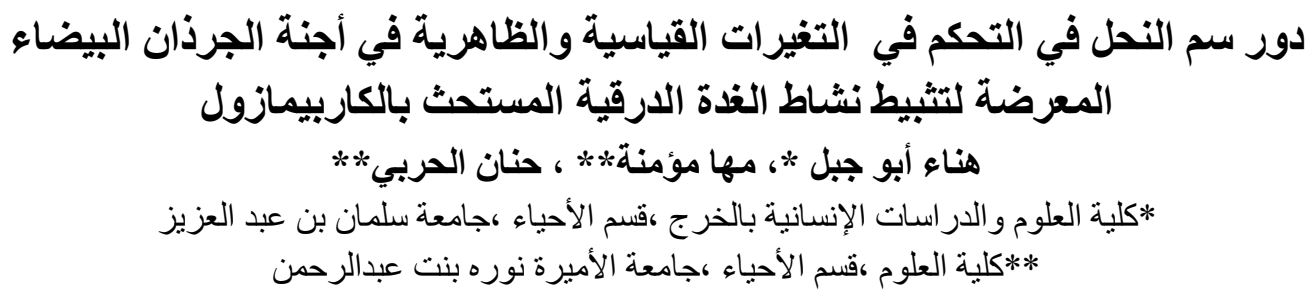

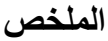

الهذف من البحث:استهدف هذا البحث دراسة دور سم النحل في التحكم في التغيرات القياسية والظاهرية التي يحدثها تثبيط

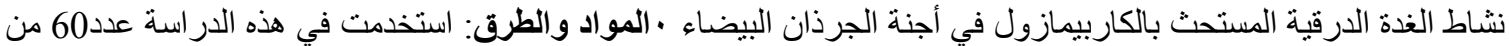
إناث الجرذان البيضاء العذارى Female Albino Rats من جنس Wistar wistar و و30 من الذكور

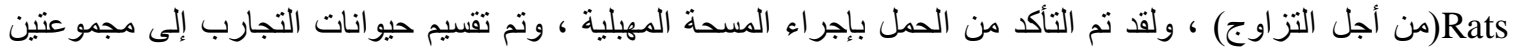
رئيسيتين :أو لاً - المجموعة التجريبية الضابطة والتي تم حقنها بالمادة المذيبة (الماء المقطر )لسم النحل و وعقار الكاربيمازول

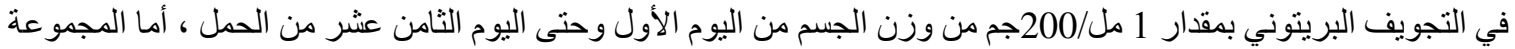

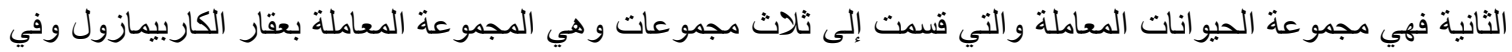

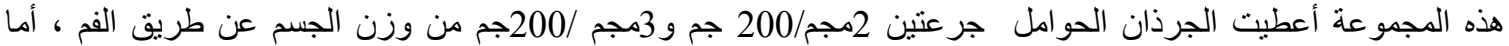

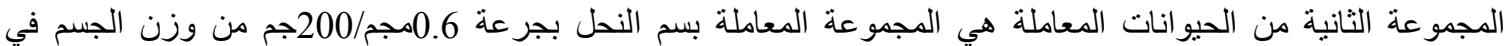

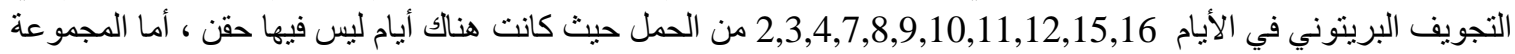
الثالثة فهي مجموعة العلاج و التي عولجت فيها الحيو انات بسم النحل بعد ساعة من الحقن بالكاربيمازول في الأيام المحددة كما

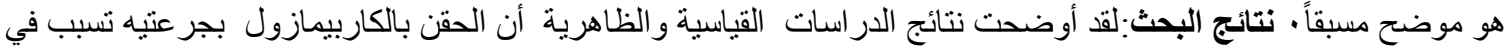

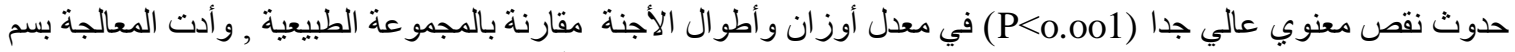

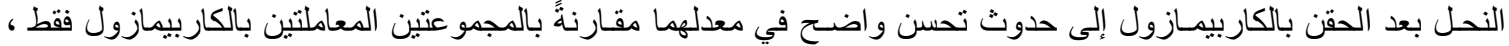

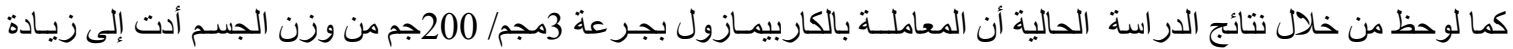

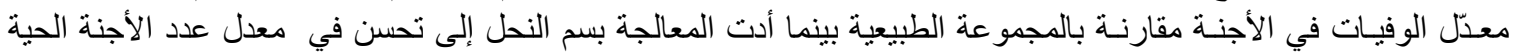

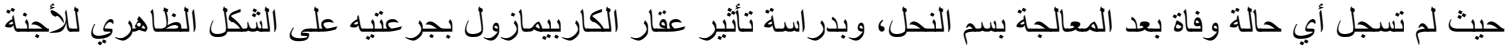

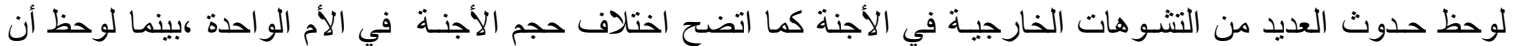

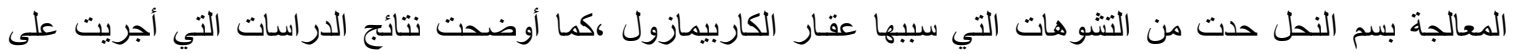

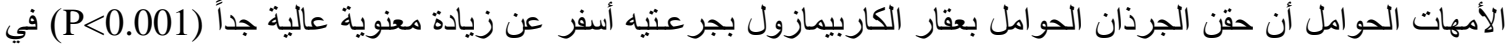

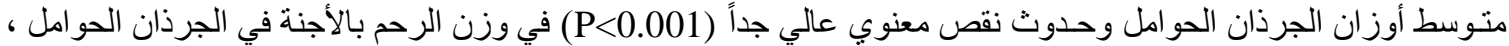

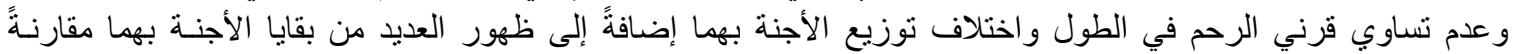
بالمجمو عة الطبيعية ، وبعد العلاج بسم النحل حدث تحسن و اضـح وملموس لهذه التغير التهات.

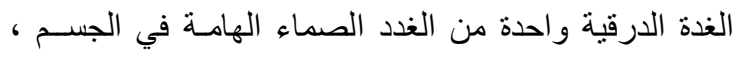

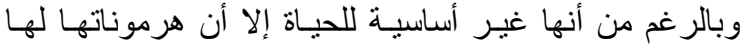

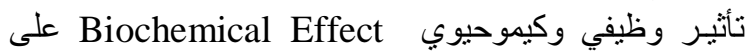

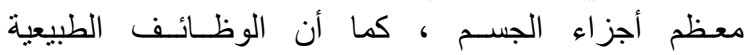

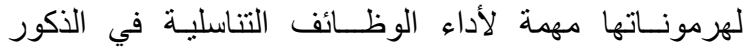

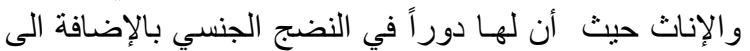

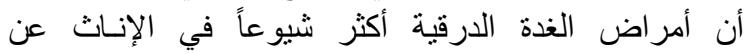

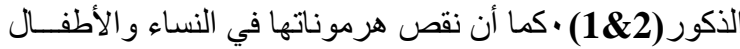

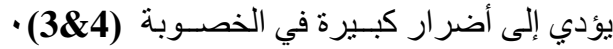

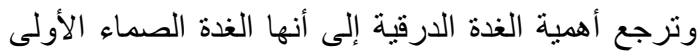
التي تتكون في جنين الإنسان ما بين الأسبوع الثالث والرابع الثابع الإلى

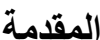

من الحمل ، وييدأ إنتاج المادة الغروية داخلها في الحويصلات

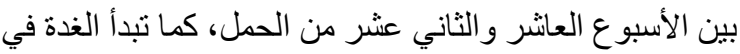
أداء وظائفها بعد الثهر الثالث وانث (5) • قد يحدث أثناء الحمل ارتفاع زائد في نشاط الغدة الدرقية وذللك

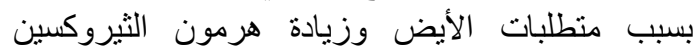
Thyroxin $\left(\mathrm{T}_{4}\right)$

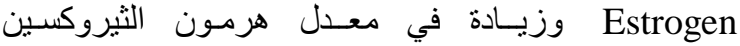
المرتبـط بالجلوبيولين (TBG) •(6\&7)Globulin وقد أوضحت الدراسات أن التغير في نشاط الغدة الدرقية في

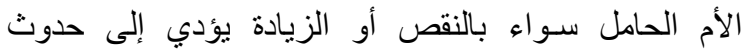

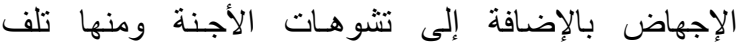




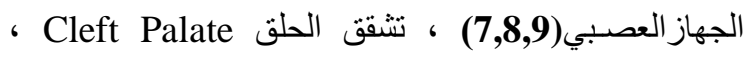

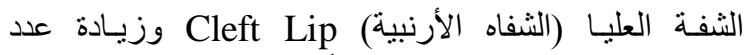
الأصابع Poly Dactyly إضـافةً إلى ولادة أطفال يعانون من مشاكل في القلب (10\&11)؛ .

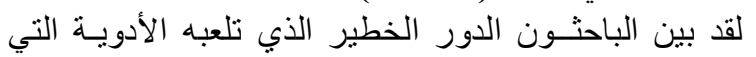

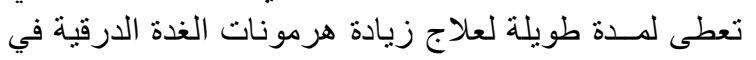

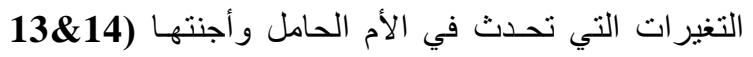
(12,

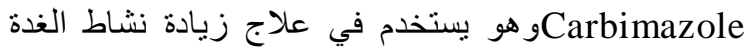

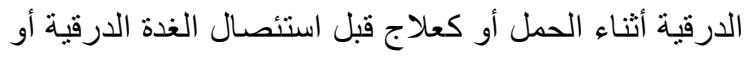

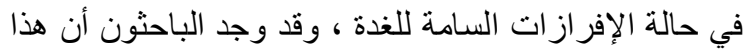

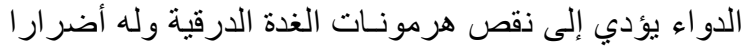

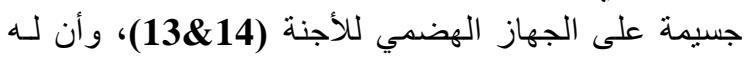

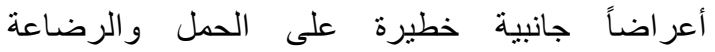

$\cdot(15,16,17)$

على الرغم من دخولنا الألفية الثالثة إلا أن الكثيرين

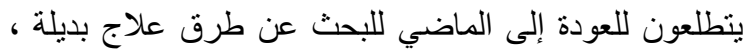

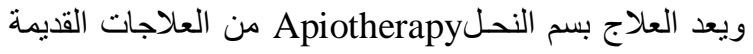

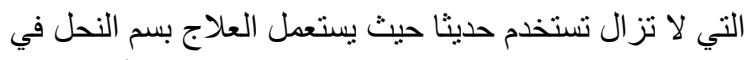

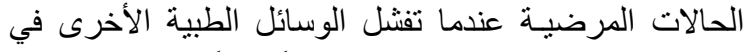

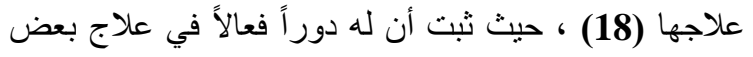

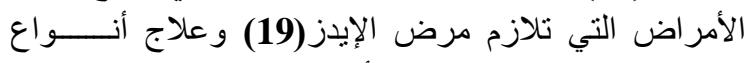

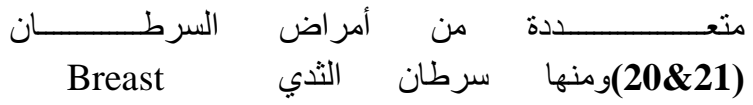
- (22\& 23)Cancer وثبت أن سـ النحل لـه فاعليـة كبــيرة جداً في تعديل

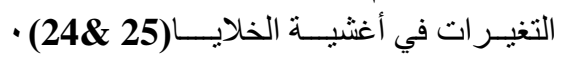

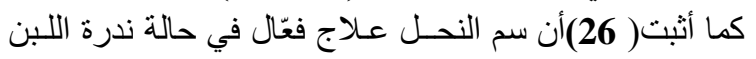

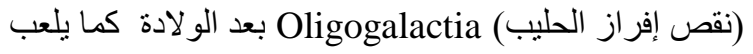

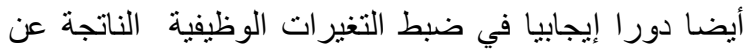
تناول الأمهات لبعض العقائيا في أثناء الحمل (27) •

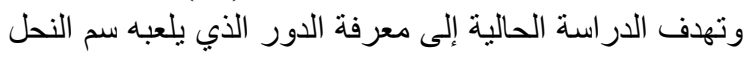

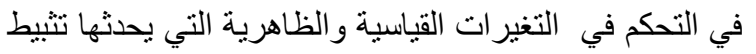
نشاط الغدة الدرقية المستحث بالكاربيمازول في أجنة الجرذان

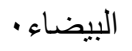

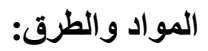

استخدمت في هذه الدراسـة إنـاث الجـرذان البيضـاء البالغــة Albino Rats

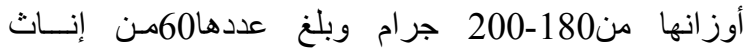

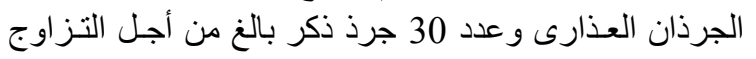

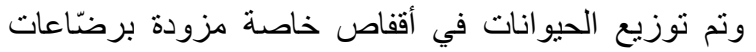

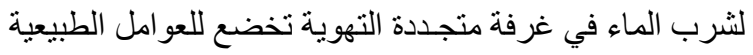

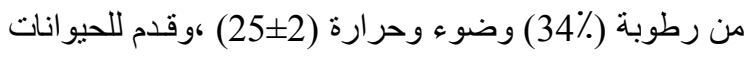

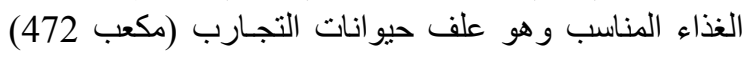

من إنتاج مصنع اراسكو للأعلاف ،كما استخدم عقار

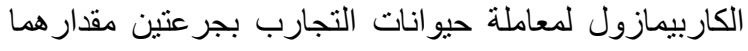

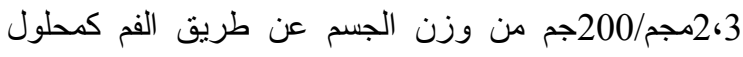

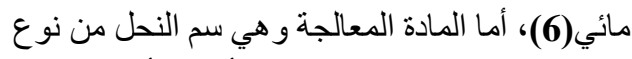
Apis Mellifera

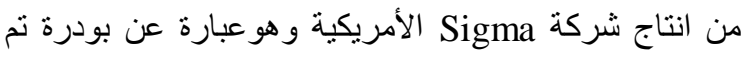

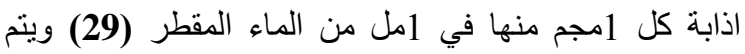

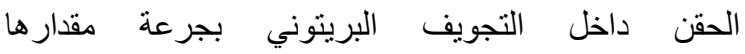
6.0مجم/200جم من وزن الجسم (30)ويتم تحديد الدورة

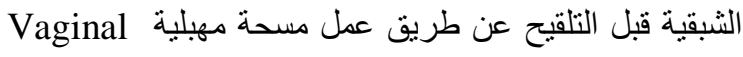
Smear الثبقية مع ذكر في أقفاص التربية طو ال الليل وفي الإن في الصباح ينت

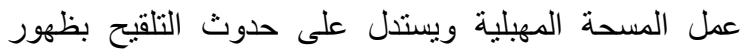

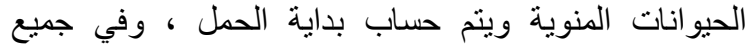

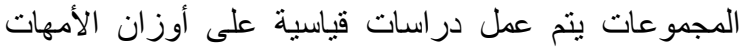

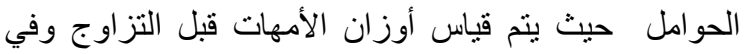

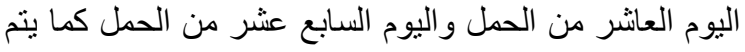

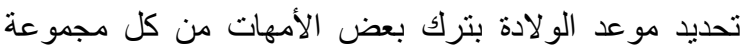

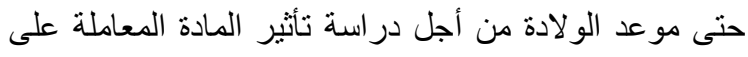

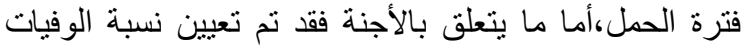

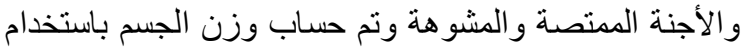

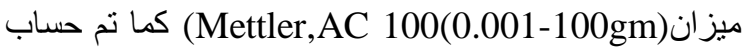
منوسط طول الجسم وذللك باستخدام مسطرة مدرجة ودر استة الثكل الظاهري للأجنة كما تم فحص الأجنة المشولة هذة فحصاً دقيقاً تصوير ها.

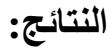

لقد أوضحت نتائج الدراسات التي أجريت على الأمهات

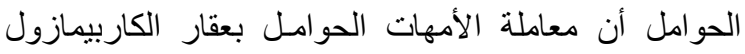

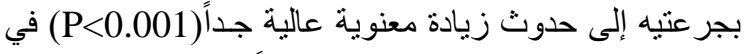

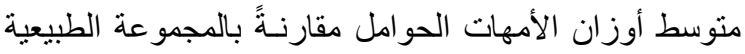

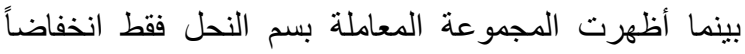

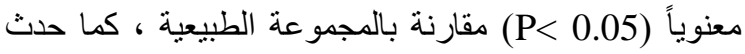

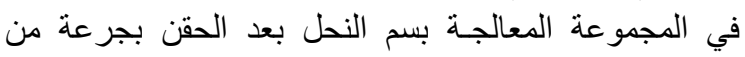

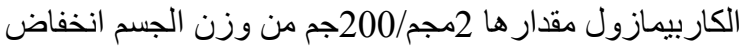

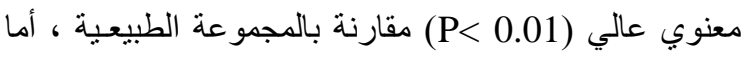

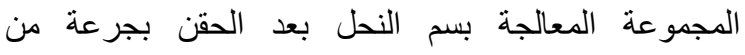

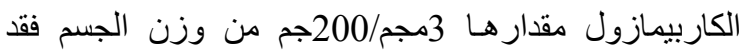

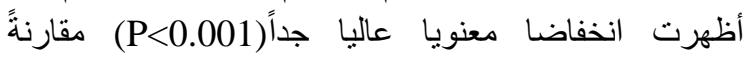

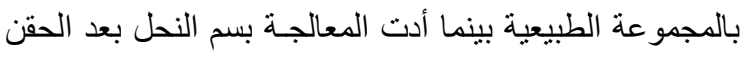

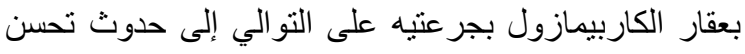

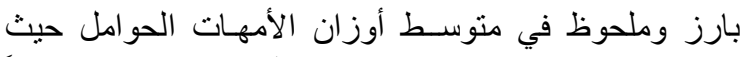

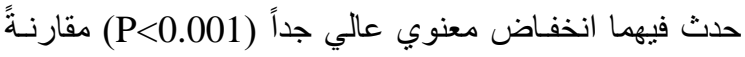

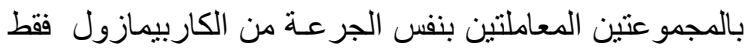
كما بتضح من جدول(1) والثكل البياني (1)، كما أشارت المعارت 
The Role of Bee Venom in Controlling the Morphometric and Morphological Changes...

النتائج أن معاملة الجرذان الحوامل بعقار الكاربيمـازول المناقشة:

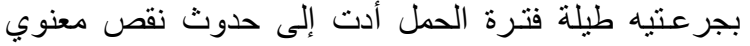

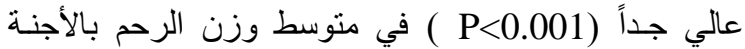

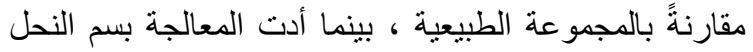

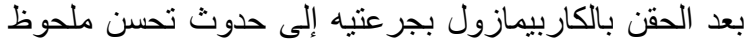

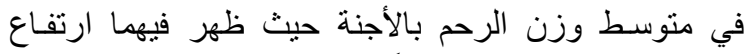

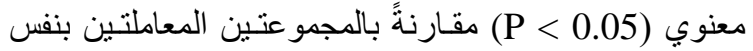

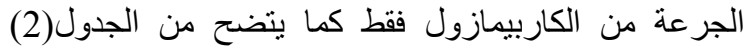

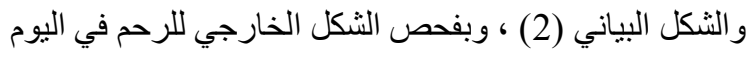

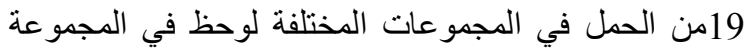

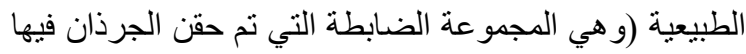

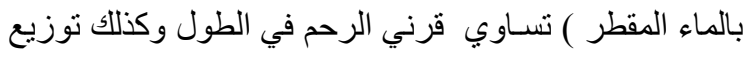

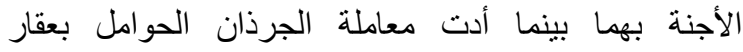

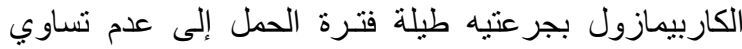

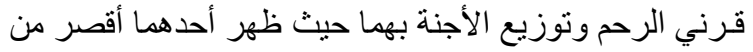

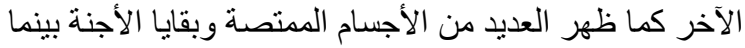

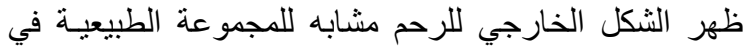

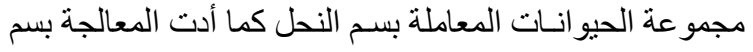

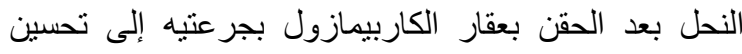

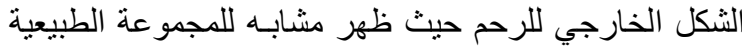

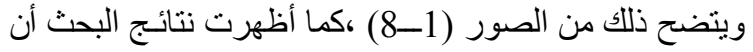

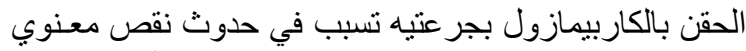

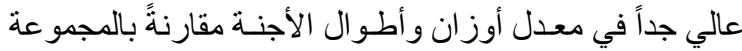

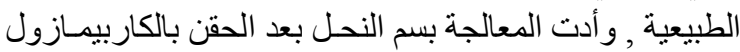

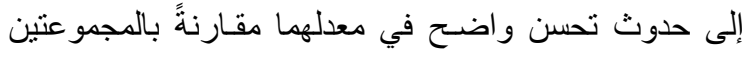

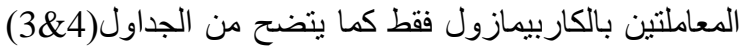

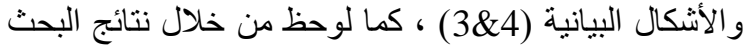

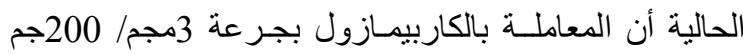

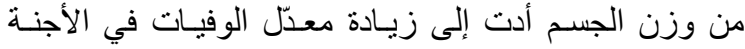

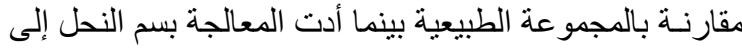

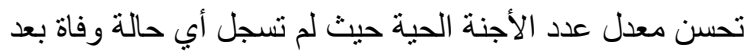
المعالجة بسم النحل ، وبدراسة تأثير عقار الكاربيمازول التئل

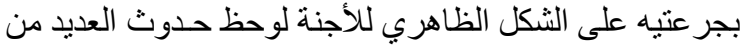

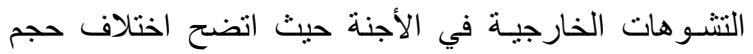

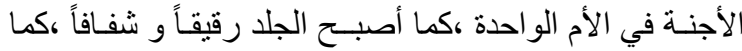
ظهرت العديد من الجلطسات الدموية تحت الجلد الجات وظهر

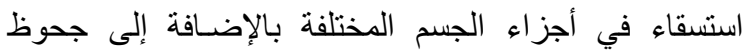
العينين وتتوه في منطقة الفم (البوز) تمنلت في ظهور الثنفة الإنة

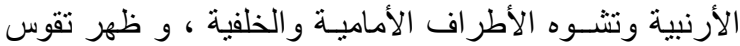

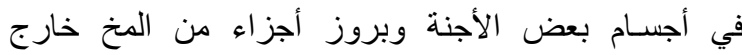

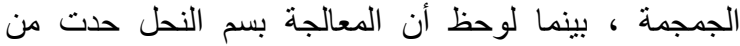
التشوهات التي سببها عقار الكاربيمازول حيث ظهرت التهات الأجنة

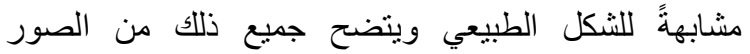
.(18-9)

تثشير نتائج الدراسة الحالية أن تثبيط هرمونات الغدة الدرقية عن طريق المعاملة بعقار الكاربيمازول طيلة فترة الحمل أدى إلى زيادة في معدل أوزان الجرذان الحوامل في كلا المجمو عتين اللتين تم حقنهما بالكاربيمازول مقارنة بالمجمو عة

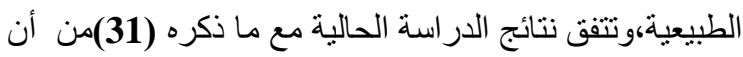
حقن الجرذان الحوامل بالكاربيمازول ينتج عنه زيادة في أوزان الجرذان، كما يؤيد ذلك( 33و32) حيث أكدا أن تثبيط نشاط الغدة الدرقية من أسباب زيادة الوزن نتيجة نقص التحكم في وظيفة الغدة الدرقية عند العلاج البديل،كما أوضح (31) أن حقن الجرذان الحوامل بالكاربيمازول أثناء فترة الحمل يؤدي إلى زيادة في معدل أوز انها نتيجة زيادة مستوى الدهون في الأنسجة واتضح ذللك من خلال صبغ الأنسجة بصبغة أسود سودان التي أوضحت المستوى العالي للاهون، كما قد يكون بطئ عمليات الأيض سبباً في زيادة الوزن ويؤيد ذلك ما أوضحسه (32و34) •ولقد أوضحا أن نقص إفراز هرمونات الغدة الدرقية يؤدي إلى نقص معدل التمثيل الأساسي في الجسم

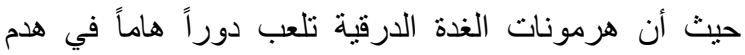

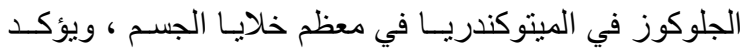
ذللك (33)حيث اتضح لهم من خلال تقرير لحالة مرضية أن القصور الدرقي يؤدي إلى انخفاض معدل التمثيل الأساسي في هن هري

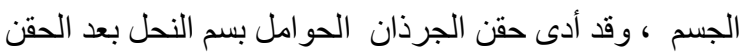
بالكاربيمازول بجر عتيه إلى عودة معدل وزن الجسم للجرذان الحوامل إلى مستواه الطبيعي مقارنةً بالمجموعة الطبيعية و المجمو عتين المعاملتين بالكار بيمازول بجر عتيه.

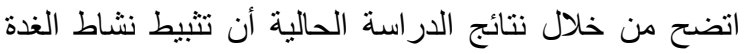
الدرقية عن طريق معاملة الجرذان الحوامل بالكاربيمازول بجر عتيه قد تسبب في انخفاض و اضح في معدل أوزان الرحم بالأجنة وعدم تساوي قرني الرحم مع عدم تساوي توزيع

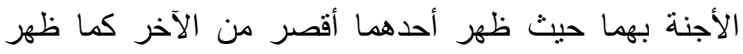
العديد من الأجسام الممنصة وبقايا الأجنة بها. و اتفق ( 35 ) مع النتائج الحالية حيث ذكرا أن إصابة النساء بالقصور الدرقي يتسبب في تغيرات عديدة في نركيب الرحم

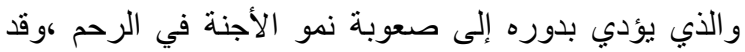




\section{Abu Gabal H et al}

برهنت (6 )أن تنبيط نشاط الغدة الدرقية في الجرذان الحوامل بالكاربيمازول تسبب في ضمور أو قصر أحد أو كلا قرني

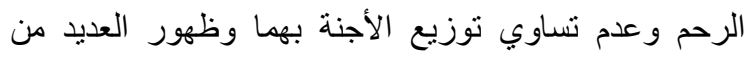

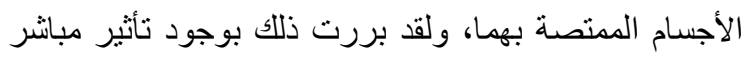
للقصور الدرقي على حدوث الاضطرابات المبيضية والتي

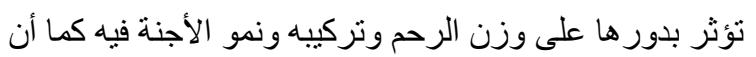

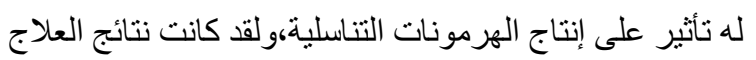
بسم النحل معنويةً في عودة وزن الرحم إلى المعدل الطبيعي

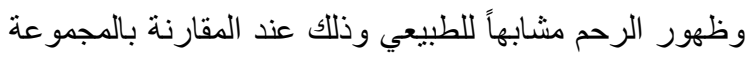
الطبيعية و المجمو عتين المعاملتين بالكاربيمازول. تتثير نتائج الدراسة الحالية إلى أن نثيط نشاط الغدة الدرقية

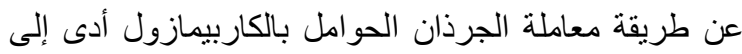
حدوث نقص معنوي عالي جداً في معدل نمو و أعداد الأجنة مقارنة بالمجموعة الطبيعية، ويتفق (36و 37 معل ) مع نتائج الدراسة الحالية والذين وجدوا أن معالجة الأم الحامل بعقار

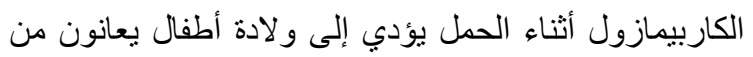

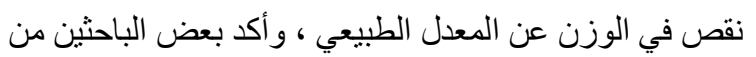

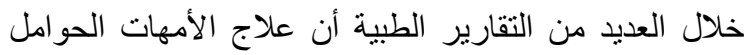

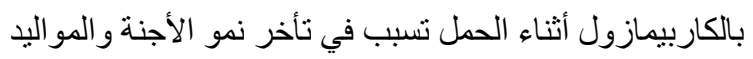
وانخفاض أوزان الأجنةوإعاقة النمو داخل الرحم (37) وأضاف(38) أن النقص في مستوى هرمونات الغدة الدرقية

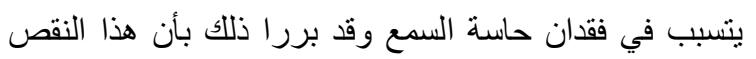
يؤدي إلى انخفاض معدل التمثيل في الجسم والذي يؤثر بدوره

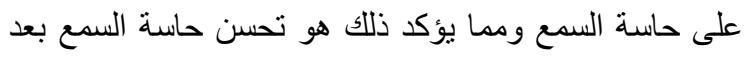
علاج النقص في مستوى هرمونات الغدة الدرقية وتحسن معدل التمثيل في الجسم .،وتتفق نتائج (6) مع تللك في الدراسة

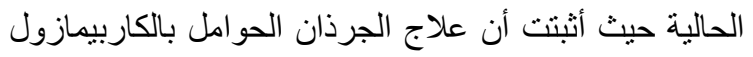
تسبب في تأخر نمو الأجنة من حيث أوزان وأطوال الأجنة

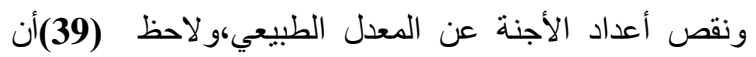
معاملة الجرذان الحوامل بالكاربيمازول تؤدي إلى انخفاض الدئ معدل أطوال الأجنة عن المعدل الطبيعي نتيجة قصر عظمتي بان باني الزند والقصبة، ومن المحتمل أن سبب نقص معدل النمو عن الطبيعي في أجنة الجرذان الحوامل و التي تم تثبيط هرموناتها عن طريق معاملتها بعقار الكاربيمازول هو نقص لهـ معدل
هرمون الثثروكسين الذي يصل إلى الجنين من الأم عبر

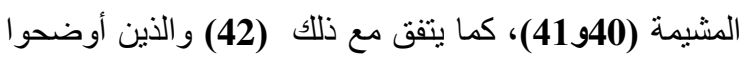
أن الجنين أثناء حياته داخل الرحم يعتمد كليةً على ما يصل إليه من الأم عبر المشيمة من مواد غذائية ومواد أيضية ومنها لهانها

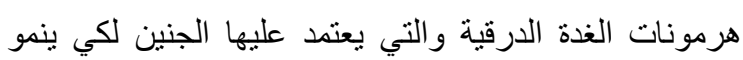
بشكل طبيعي و هذه الهرمونات يحتاج إليها الجنين خلال الفترة

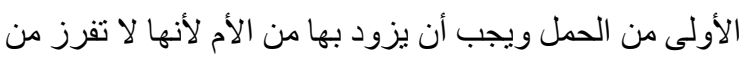

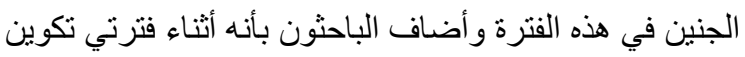
الأعضاء و اكتمال الجنين سوف يعتمد الجنين على ما ينتقل إليه

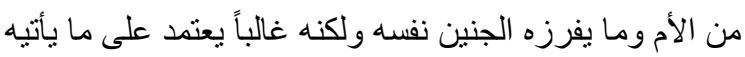

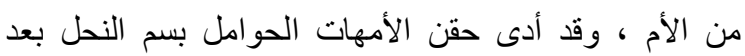

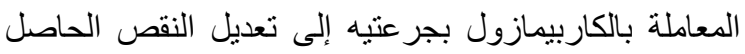

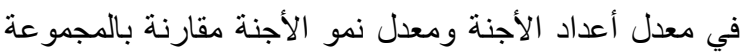
الطبيعية والمجمو عتان المعاملتان بالكاربيمازول بجر عتيه. تشير نتائج الدراسة الحالية إلى أن حقن الجرذان الحوامل بالكان

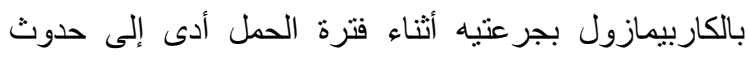
العديد من التشوهات في الأجنة مقارنةً بالمجموعة الطبيعية،

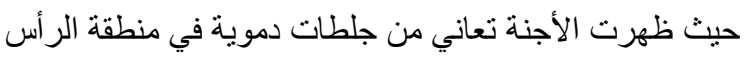
والأطراف و البطن، كما ظهر الجلد رقيقاً واكتسب لوناً باهناً

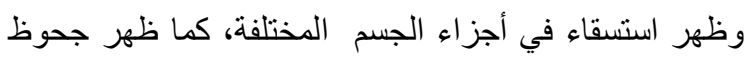
في العينين وتتشوه منطقة الفم وتثوه في الأطراف الأمامية

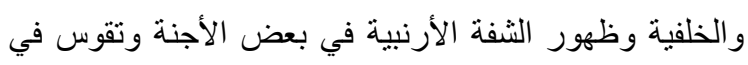

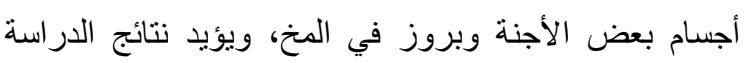
الحالية (36) والذي أوضح أن علاج الأمهات الحوامل فئل فئل بالكاربيمازول تسبب في العديد من نتشوهات الأجنة ومنها

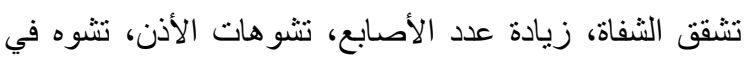

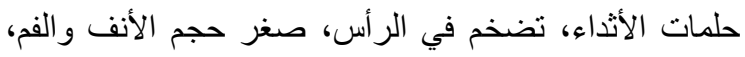

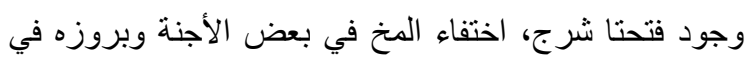
البعض الآخر وحدوث خلل في الجهاز العصبي، ويتفق معنا (43) و الذين أثنتوا أن علاج الأمهات الحوامل بالكاربيمازول يتسبب في حدوث العديد من التشوهات الخلقية في الأجنة ومنها تلف جدار البطن حيث يؤدي إلى الفتق السري وبروز الأحشاء

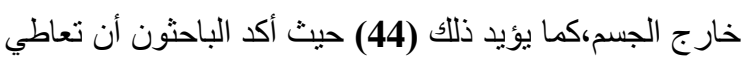

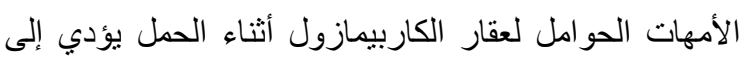


The Role of Bee Venom in Controlling the Morphometric and Morphological Changes...

حدوث العديد من التشوهات للأجنة فقد لاحظ الباحثون

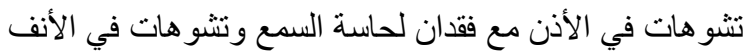
، تشوه الأصابع إضافةً إلى تغير ملامح الوجه عن الثنكل

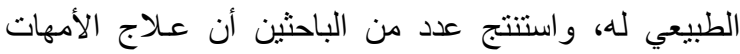
الحو امـل بالكاربيهـازول يؤدي إلى حدوث خلل في ملونـات

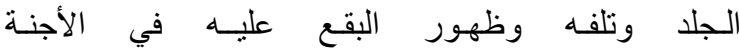
$\cdot(47946945938)$

كما أوضح عدد من الباحثين أن القصور الدرقي يسبب نقص في معدل نمو المخ في بعض الأجنة وضموره في البعض

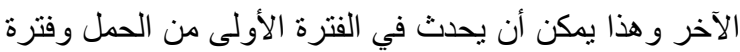
تكوين الأعضاء (41و48و49) ،هذا وقد برر ( 41 )ما سبق بنقص ما يصل إلى المخ من هرمون Th, و أكد (48 ) أن اضطر اب مستوى هرمونات الغدة الدرقية يؤدي إلى خلل في عمليات الأيض في المخ والتي تتخخل بها الخلابا النجمية Astrocyte الموجودة في النسيج الضام كما أنها

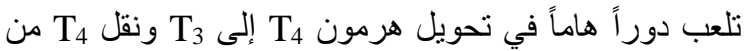
الدم إلى أنسجة المخ ، كما أثبت بعض الباحثين أن العلاج

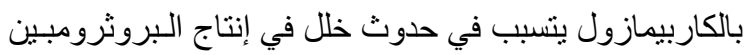
وزيـادة إنتاجه مهـ يتسبب في هدوث الجلطـات

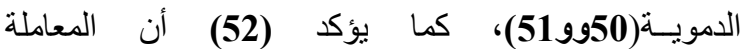
بالميركازول Mercazole أدت إلى زيادة معدل الطفرات في

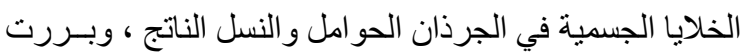
(6) حدوث تشوهات الأجنة نتيجة معاملة الجرذان الحوامل بالكاربيمازول إلى العدد المتز ايد من الخلايا الميتة خلال الفترة الحساسة لنمو الجنين ، داخل الرحم وهي فترة تكوين الأعضــاء ، وقد أدى حقن الأمهات الحوامل بسم النحل بعد الحقن بالكاربيمازول بجرعتيه إلى الحد من التشوهات التي

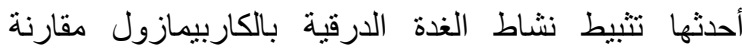
بالمجموعة الطبيعية والمجموعتان المعاملتان بالكاربيمازول

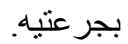
كما أثنتت نتائج الدراسة الحالية الدور الكبير والفعال الذي يلعبه سم النحل في تعديل التغيرات السلبية التي تسبب بها تثبيط نثاط الغدة الدرقية عن طريق استخدام عقار الكاربيمازول فكان أثر العلاج به واضحاً سواء كان ذللك في التحكم في
التغيرات الوظيفية أو التغيرات في التركيب النسيجي ومن ثم دوره الكبير في الحفاظ على الثكل الظاهري لأجنة الأمهات المعالجة به كما هو في المجموعة الطبيعية، ويتفق مع نتائج الدراسة الحالية في الدور الذي بلعبه سم النحل كعلاج بديــــلـل

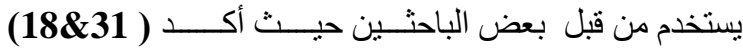
أن سم النحل ليس له أي آثار جانبية على جميع أجهزة الجسم

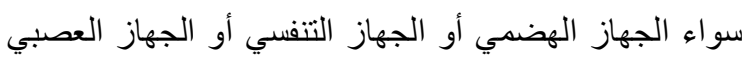

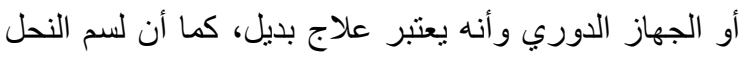
فاعلية كبيرة جداً في تعديل التغيرات في أغثــية الخلايـا (25\&26)، وقد برر(24) الدور الذي يلعبه سم النحل في تعديل التغيرات في أغثية الخلايا بتعديل تفاعلات البروتينات الدهنية التي تدخل في تكوين هذه الأغشية وذلك لوجود مادة الميليتين في تركيبه، أما الباحثان(25) فقد عز ا تعديل تفاعلات البروتينات الدهنية إلى وجود إنزيم الفوسفوليباز في تركيب سم

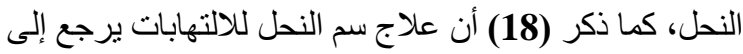

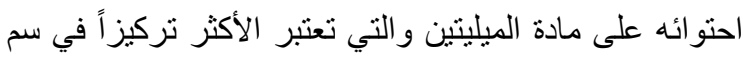
النحل ولها من الفعالية ضد الالتهابات ما يقدر بـ 100 مرة ضyd فعالية مادة هايدروكورتيزول Hydrocortisol و أضاف الباحث أن سم النحل يلعب دوراً فعالاً في التخفيف من هن

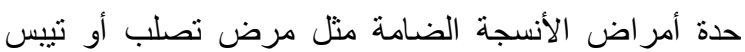
طبقات الجلد وأمر اض تليف العضلات(53\&54) وقد برر ذلك بأن سم النحل يعمل على تحفيز الفص الأمامي من الغدة ولذان

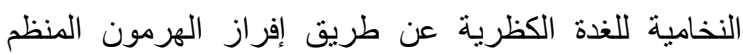
لقشرة الغدة الكظريـة Adreno - Cortico (ACTH) ويطلق عليه كورتيكوتروبين Tropic Hormone Corticotropin لإفراز هرموناتها ومن أهمها الكورتيزون الذي بلعب دوراً

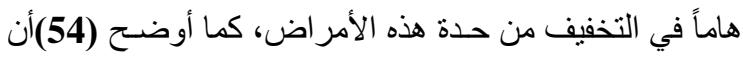
يؤثر على النسيج الدهني فيسهل عملية تحليل (ACTH) الدهون وتحويلها إلى أحماض دهنية وبالتالي فإنه من المحتمل أن يكون هذا سبب دور سم النحل في انخفاض الوزن في الأمهات الحوامل وعودته إلى المعدل الطبيعي كما هو في المجموعة الضابطة كردة فعل معاكسة لما يقوم به عقار الكاربيمازول من ضمور للغدة الكظرية وزيادة تخزين الدهون 


$$
\begin{aligned}
& \text { مما أدى إلى ارتفاع الوزن عن المعدل الطبيعي في الأمهات }
\end{aligned}
$$

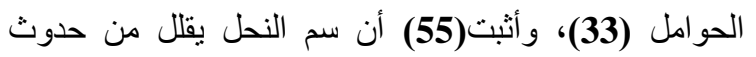

$$
\begin{aligned}
& \text { الجلطات ويؤيد ذلك ما أكده (56)من أن سم النحل يؤدي إلى إنى } \\
& \text { نقص مادة البروثرومبين الهامة في حدوث عملية التجلط } \\
& \text { وبالتالي فإن هذا يؤيد ما تم قوله عن الدور الذي يلعبه سم } \\
& \text { النحل في تعديل التغيرات التي قام بها عقار الكاربيمازول } \\
& \text { والتي منها حدوث الجلطات الناتجة عن خلل في إنتاج } \\
& \text { البروثرومبين وزيادة إنتاجه وبالتالي حدوث العديد من } \\
& \text { الجلطات الدموية(50\&51)، مما يؤكد فاعلية سم النحل كعلاج }
\end{aligned}
$$

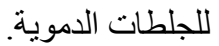

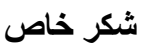

$$
\begin{aligned}
& \text { هذا البحث مدعم من مدينة الملك عبد العزيز للعلوم و التقنية }
\end{aligned}
$$

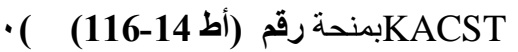

\section{References:}

1- Dittrich R, Beckmann MW, Oppelt PG, Hoffmann I, Lotz L, Kuwert T and Mueller A (2011): Thyroid hormone receptors and reproduction.J . Reprod. Immunol., 90(1):58-66.

2- Santin A P and Furlanetto T W (2011): Role of estrogen in thyroid function and growth regulation.J .Thyroid Res. ,2011: 1-7.

3- Alkafajei A, Amarin Z, Alazaizeh W, Khader Y and Marji M (2012): Prevalence and risk factors for hypothyroidism in Jordanian women: comparison between different reference ranges. $\mathrm{J}$.East Mediterr. Health,18 (2): 132-136.

4- Artini P, Uccelli A, Papini F, Simi G, Di Berardino O, Ruggiero $M$ and Cela V (2013) : Infertility and pregnancy loss in euthyroid women with thyroid autoimmunity.Gynecol. Endocrinol., 29(1): 36-41.

5- Mansourian A (2010): Thyroid function tests during first-trimester of pregnancy: A Review of Literature.

Pakistan Journal of Biological Sciences, 13: 664-673 6-Fawzy M (2002): Increased risk of fetal anomalies following maternally induced hypothyroidism in female albino rats.Ph.D. Thesis, Faculty of Science, Cairo Univ.

\section{7-Patel J, Landers K, Li H, Mortimer RH and}

Richard K.(2011 ): Thyroid hormones and fetal neurological development.J. Endocrinol , 209(1 ):18 .

8- Roepke T, King E, Reyna-Neyra A, Paroder M, Purtell K, Koba W, Fine $\mathbf{E}$ and Abbott
G(2009): Kcne2 deletion uncovers its crucial role in thyroid hormone biosynthesis. Nature Medicine, 15: $1186-1194$.

\section{9- Jordi J, Mar A, Marisa R, Mario M, Joan F} and Raquel G (2013): Thyroxine levels during pregnancy in healthy women and early child neurodevelopment. Epidemiol .,24 (1) 150157.

10- Khochtali I, Hamza N, Harzallah O, Hamdi S, Saad J, Golli M and Mahjoub S (2011) : Reversible dilated cardiomyopathy caused by hypothyroidism.Int. Arch Med., 2011:4-20.

11- Kota $S$,Ranjan $P$, Kota $S$, Jammula $S$, Meher L and Modi K (2012): Primary hypothyroidism: uncommon presentation with reversible dilated cardiomyopathy in young subjects.J. Endocrinol.Metab., 10:440-443.

12-Chakraborty G, Magagna-Poveda A, Parratt C, Umans JG, MacLusky $N$ and Scharfman H (2012): Reduced hippocampal brain-derived neurotrophic factor (BDNF) in neonatal rats after prenatal exposure to propylthiouracil (PTU).

Endocrinol. ,153(3):1311-1316.

13 -Koenig D , Spreux A, Hiéronimus S, Chichmanian RM, Bastiani F, Fénichel $P$ and Brucker-Davis F (2010): Birth defects observed with maternal carbimazole treatment:

Six cases reported to Nice's Pharmacovigilance Center.

Ann .Endocrinol. (Paris), 71(6):535-542.

14 - De Swiet M (2002): Medical Disorders.Obst. Practice, $4^{\text {th }}$ ed. Oxford: Blackwell Science.

15 - Diav-Citrin $O$ and Ornoy A (2002): Teratogen update: antithyroid drugs methimazole, carbimazole and propylthiouracil.Teratol., 65:38.

16- McElbatton $P$ (2002): Teratogenic drugs- part 2.Adverse Drug Reactions.Bulletin, 214:819- 822. 17- Bowman P, Osborne NJ, Sturley R, Vaidya B.(2012): Carbimazole embryopathy: implications for the choice of antithyroid drugs in pregnancy.Q.J.M., 105(2):189-193.

18- Teichfischer P (2010): Investigations about concept and history of apitherapy-a contribution to complementary alternative medicine.

Wurzbg. Medizinhist. Mitt ., 29:278-313.

19- Trindade RA, Kiyohara PK, de Araujo PS and Bueno da Costa MH (2012):

PLGA microspheres containing bee venom proteins for preventive immunotherapy.

Int. J. Pharm., 423(1):124-33.

20- Jo M, Park MH, Kollipara PS, An BJ, Song HS, Han SB, Kim JH, Song MJ and Hong JT 
The Role of Bee Venom in Controlling the Morphometric and Morphological Changes...

(2012):Anti-cancer effect of bee venom toxin and melittin in ovarian cancer cells through induction of death receptors and inhibition of JAK2/STAT3 pathway.

Toxicol. Appl. Pharmacol., 258(1):72-81.

21- Ip SW, Chu YL, Yu CS, Chen PY, Ho

HC, Yang JS, Huang HY, Chueh FS, Lai TY and Chung JG(2012):Bee venom induces apoptosis through intracellular $\mathrm{Ca} 2+$-modulated intrinsic death pathway in human bladder cancer cells.

Int .J .Urol., 19(1):61-70.

22- Son DJ, Lee JW, Lee YH, Song HS, Lee CK and Hong JT (2007): Therapeutic application of anti-arthritis, pain-releasing, and anti-cancer effects of bee venom and its constituent compounds.Pharmacol. Ther., 115(2):246-270.

23- SIU-WAN IP, SHIN-SHIN L ,SHUW-YUAN $L$,JING-PIN $L$,JAI-SING $Y$ and MENGLIANG $L$ (2008): The role of mitochondria in bee venom-induced apoptosis in human breast cancer MCF7 cells. In vivo, 22 (2): 237-245.

24- Bollinger J, Diraviyam K, Ghomashchi F, Murray D and Gelb M (2004): Interfacial binding of bee venom secreted phospholipase A2 to membranes occurs predominantly by a nonelectrostatic mechanism.Biochemisry, 43(42):13293-13304.

25- Raghuraman $H$ and Chattopadhyay A (2004): Influence of lipid unsaturation on membrane-bound melittin: a fluorescence approach.Biochem.Biophys.Acta, 1665(1-2):29-39.

26- Choi S, Kang S, Bae C, Cho S and Pak S (2003): Effect of bee venom treatment in sows with oligogalactic syndrome

postpartum.Am.J.Chin.Med., 31(1):149-155.

27- Abu Gabal H, Moamena $M$ and Al Moalla $H$ (2012): The role of bee venom in ameliorating the physiological changes of carbimazole induced hypothyroidism in pregnant rats. The Egyptian Journal of Hospital Medicine, 49: 975-985.

28-Dombrowski $\quad$ Y, Peric $\quad$ M, Koglin

S, Kaymakanov N, Schmezer V, Reinholz

M, Ruzicka T and Schauber J ( 2012): Honey bee (Apis mellifera) venom induces AIM2 inflammasome activation in human keratinocytes.Allergy, 67(11):1400-1407.

29- Kim H , Kwon Y, Ham T , Roh D ,Yoon S , Kang S, Yang I , Han H, Lee H, Beitz A and Lee J (2004) : General pharmacological profiles of bee venom and its water soluble fractions in rodent models.J.Vet.Sci.,5(4):309-318 .
30- Liu X, Chen D, Xie L and Zhang R (2002): Effect of honey bee venom on proliferation of $\mathrm{K}$ $1735 \mathrm{M}_{2}$ mouse melanoma cells in vitro and growth of murine B 16 melanomas in vivo.

J. Pharmacy and Pharmacol., 54: 1083-1089.

31- Brunova J, Bruna J, Joubert $G$ and Koning M (2003): Weight gain in patients after therapy for hyperthyroidism.S.Afr. Med.J., 93(7):515-516.

32-Strollo F , Carucci M, Morè G, Marico G , Strollo, M. A. Masini, and S. Gentile (2012):Free Triiodothyronine and Cholesterol Levels in Euthyroid Elderly T2DM Patients. Int J Endocrinol. (Pubmed a head of print).

33-Dutta P, Bhansali A, Walia R, Khandelwal N, Das S and Masoodi SR (2012):Weight homeostasis \& its modulators in hyperthyroidism before \& after treatment with carbimazole.

Indian J .Med. Res., 136(2):242-248.

34- Jde J, Alvirde-Garcia U, López-Carrasco G, Padilla Mendoza ME, Mehta R, ArellanoCampos O, Choza R, Sauque L, Garay-Sevilla ME,Malacara JM, Gomez-Perez FJ and AguilarSalinas CA.(2010): TSH and free thyroxin concentrations are associated with differing metabolic markers in euthyroid subjects.

Eur. J. Endocrinol., 163(2):273-278.

35- Inuwa IM and Williams MA (2006): A morphometric study on the endometrium of rat uterus in hypothyroid and thyroxin treated hypothyroid rats.

Ups .J. Med. Sci.,11(2):215-225.

36- Anne-Maëlle $G$, Michel $P$ and Pierre $S$ (2003): Carbimazole-related gastroschisis. Ann. Pharmacother., 37(6):829-831.

37-Su P, Huang K, Hao J, Xu Y, Yan S, Li T and Tao F (2011): Maternal thyroid function in the first twenty weeks of pregnancy and subsequent fetal and infant development: A prospective population-based cohort study in China .J. Clin. Endocrinol. Metab., 96: 3234-3241.

38- Comer D and McConnell E (2006): Hypothyroid associated deafness.Endocrin.Abstracts, 12:124-130.

39- Ahmed $M$ and Janjua $Z$ (2003): Effect of hypothyroidism and thyroxin replacement on growth of long bones in prenatally treated albino rats.J.Pak. Med. Assoc., 53(1):18-21.

40- Julian B, Grenville F, Jonathan $\mathbf{R}$ and Jonathan B (2002): Choanal atresia: the result of maternal thyrotoxicosis or fetal carbimazole.Am.J.Med.Genet., 111(1):55-56. 
41- Dugal T, Mcentagart $M$ and Albanese $A$ (2006): Tri-iodothyronine, how does it get in? What if it goes wrong? Endocrinol.Abstracts, 12:135-140.

42- Mondal S, Pathak $M$ and Varshney V (2006): Peripheral plasma thyroid hormone concentrations during pregnancy in black Bengal goats.Endocrinol.Abstracts, 12:100-106.

43- Nicola F, Ian W, Frances $E$ and Sahar $M$ (2005): Carbimazole embryopathy: An emerging phenotype.Am.J.Med. Genetics, 132: 130-135.

44- Martin-Denavit T, Edery P, Plauchu H, Attia -Sopol J, Jaudrant D, Aurand J and Thomas L (2000): Ectodermal abnormalities associated with methimazole intrauterine exposure

. Am.J.Med. Genet., 94:338-340.

45- Karlsson F, Axelsson $O$ and Melhus H (2002): Severe embryopathy and exposure to methimazole in early pregnancy.J.Clin.Endocrinol. Metab., 87:946-949.

46-Fang Q, Giordimaina A, . Dolan D, Camper S, and Mustapha M (2012): Genetic Background of Propldf Mutants Provides Remarkable Protection against Hypothyroidism-Induced Hearing Impairment.J .Assoc .Res .Otolaryngol. 13(2): 173184.

47- Glinoer D (2001): Potential consequences of maternal hypothyroidism on the offspring: evidence and implications. Horm. Res., 55(3):109-114.

48- Trentin AG (2006): Thyroid hormone and astrocyte morphogenesis.J. Endocrinol., 189(2):18997.

49- Kontoleon P, Ilias I ,Koutras D, Kontogiannis D and Papapetrou P (2002): Successful treatment with carbimazole of a hyperthyroid pregnancy with hepatic impairment after propylthiouracil administration: a case report.Clin. Exp.Obstet. Gynecol., 29(4):304-305.
50- Khoo A, Tham L, Lee K and Lim G (2003): Acute liver failure with concurrent bupropion and carbimazole therapy. Ann. Pharmacother., 37(2):220-223.

51- Bux J, Ernest-Schlegel $M$, Rothe $B$ and Panser C (2000): Neutropenia and anemia Due to carbmazole-dependent antibodies.Br.J.Haematol., 109(1):243-247.

52-Konoplia $E$, Nikolaevich $L$,Sechko $L$ and Glazko L (2000): The effect of acute external gamma- irradiation and thyroid glands dysfunction on rat reproductive system and frequency of structural mutations in somatic cells of pregnant females and their offsprings.Radiat.Biol.Radioecol.,40(1):43- 48.

53- Ganong W (2003): Review of Medical Physiology. $24^{\text {th }}$ ed.

Appleton and Lange, Norwalk, Connecticut / LOS Altos, California, pp: 421-442.

54- Sarwar G and Janjua $M$ (2003) : Effects of carbimazole on morphology of rat adrenals.J.Coll.Physicians Surg.Pak., 13(2):94-97.

55- Krotz F, Riexinger T, Buerkle M , Nithipatikom K ,Gloe T , Sohn H, Campbell E and Pohl U (2004): Membrane-Potential-Dependent inhibition of platelet adhesion to endothelia cells by epoxyeicosatrienoic acids .Arterioscler. Thromb.Vasc.Biol. ,24(3): 595-600.

56- Gawlik R, Rymarczyk B and Rogala B (2004): A rare case of intravascular coagulation after honey bee sting.J.Investig. Allergol. Clin. Immunol., 14(3):250-252.

جدول(1):متوسط معدل الزيادة في أوزان الأمهات الحوامل (جم) (خلال فترات مختلفة من الحمل) في المجموعات المختلفة

\begin{tabular}{|c|c|c|c|c|c|c|}
\hline $\begin{array}{l}\text { سماربيمازول النحل+ } \\
\text { (3مجم) }\end{array}$ & $\begin{array}{l}\text { سماربيمازول النحل+ } \\
\text { الكمجم) }\end{array}$ & المعاملة بسم & $\begin{array}{l}\text { 3الكاربيمازول المعاملة } \\
\text { (مجم) }\end{array}$ & $\begin{array}{l}\text { بالكاربيمازول المعاملة } \\
\text { (2مجم) }\end{array}$ & (المجموعة التي & المجموعات \\
\hline 214 & 218 & 219 & 247 & 255 & 225 & المتوسط \\
\hline $1.167 \pm * * *$ & $1.500 \pm * *$ & $2.00 \pm *$ & $1.600 \pm * * *$ & $1.770 \pm * * *$ & $1.116 \pm$ & الخطأ القياسي \\
\hline
\end{tabular}


The Role of Bee Venom in Controlling the Morphometric and Morphological Changes...

جدول(2): متوسطوزن الرحم بالأجنة (جم) في اليوم 19من الحمل للمجموعات المختلفة في أمهات الجرذان الحوامل

\begin{tabular}{|c|c|c|c|c|c|c|}
\hline $\begin{array}{l}\text { سم الكاربيمازول } \\
\text { (3مجم) }\end{array}$ & الكاربيمازول النحل+ & المعاملة بسم & $\begin{array}{l}\text { بالكاربيمازول } \\
\text { (المعاملة } 3 \text { (3جم) }\end{array}$ & $\begin{array}{l}\text { بالكاربيمازول المعاملة } \\
\text { (2مجم) }\end{array}$ & (المجموعة التبيعية & المجموعات \\
\hline 46.55 & 50.50 & 51.59 & 40.15 & 43.22 & 55.14 & المتوسط \\
\hline $\begin{array}{ll}* * & 1.883 \pm\end{array}$ & $0.667 \pm$ & $1.768 \pm$ & $1.683 \pm * * *$ & $2.349 \pm * * *$ & $2.343 \pm$ & الخطأ القياسي \\
\hline
\end{tabular}

جدول(3):متوسط أوزان الأجنة(جم) في اليوم 19من الحمل للمجموعات المختلفة في أمهات الجرذان الحوامل

\begin{tabular}{|c|c|c|c|c|c|c|}
\hline $\begin{array}{l}\text { سم الكاربيمازول } \\
\text { (3مجم) }\end{array}$ & $\begin{array}{l}\text { سماربيمازول النحل+ } \\
\text { (2مجم) }\end{array}$ & المعاملة بسم & 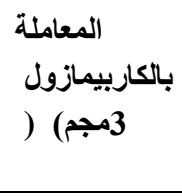 & $\begin{array}{l}\text { بالكاربيمازول المعاملة } \\
\text { (2مجم) }\end{array}$ & 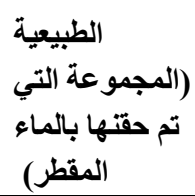 & المجموعات \\
\hline 3.3 & 3.8 & 3.4 & 1.8 & 2.9 & 4.5 & المتوسط \\
\hline $0.152 \pm * * *$ & $0.0458 \pm * * *$ & $0.153 \pm * * *$ & $0.076 \pm * * *$ & $0.157 \pm * * *$ & $0.114 \pm$ & الخطأ القياسي \\
\hline
\end{tabular}

جدول(4):يوضح متوسط أطوال الأجنة(سم) في اليوم 19من الحمل للمجموعات المختلفة في أمهات الجرذان الحوامل

\begin{tabular}{|c|c|c|c|c|c|c|}
\hline $\begin{array}{l}\text { سم الكاربيمازول } \\
\text { (3مجم) }\end{array}$ & $\begin{array}{l}\text { الكاربيمازول النحل+ } \\
\text { (2مجم) }\end{array}$ & بسم النحل & $\begin{array}{l}\text { 3الكاربيمازول المعاملة } \\
\text { (مجم) }\end{array}$ & $\begin{array}{l}\text { بالكاربيمازول المعاملة } \\
\text { (2مجم) }\end{array}$ & 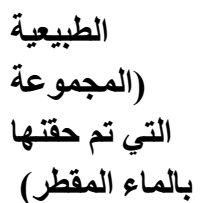 & المجموعات \\
\hline 3 & 3.2 & 3.4 & 1.6 & 2.9 & 3.9 & المتوسط \\
\hline $0.235 \pm * *$ & $* 0.209 \pm$ & $* 0.144 \pm$ & $0.183 \pm * * *$ & $0.221 \pm * * *$ & $0.0221 \pm$ & الخطأ القياسي \\
\hline
\end{tabular}

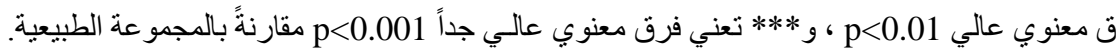

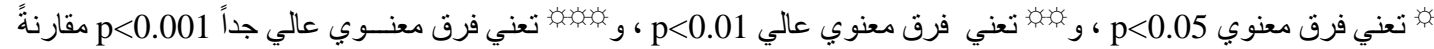

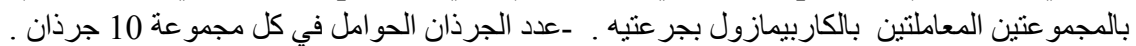

شكل (1):متوسط أوزان الأمهات الحوامل 


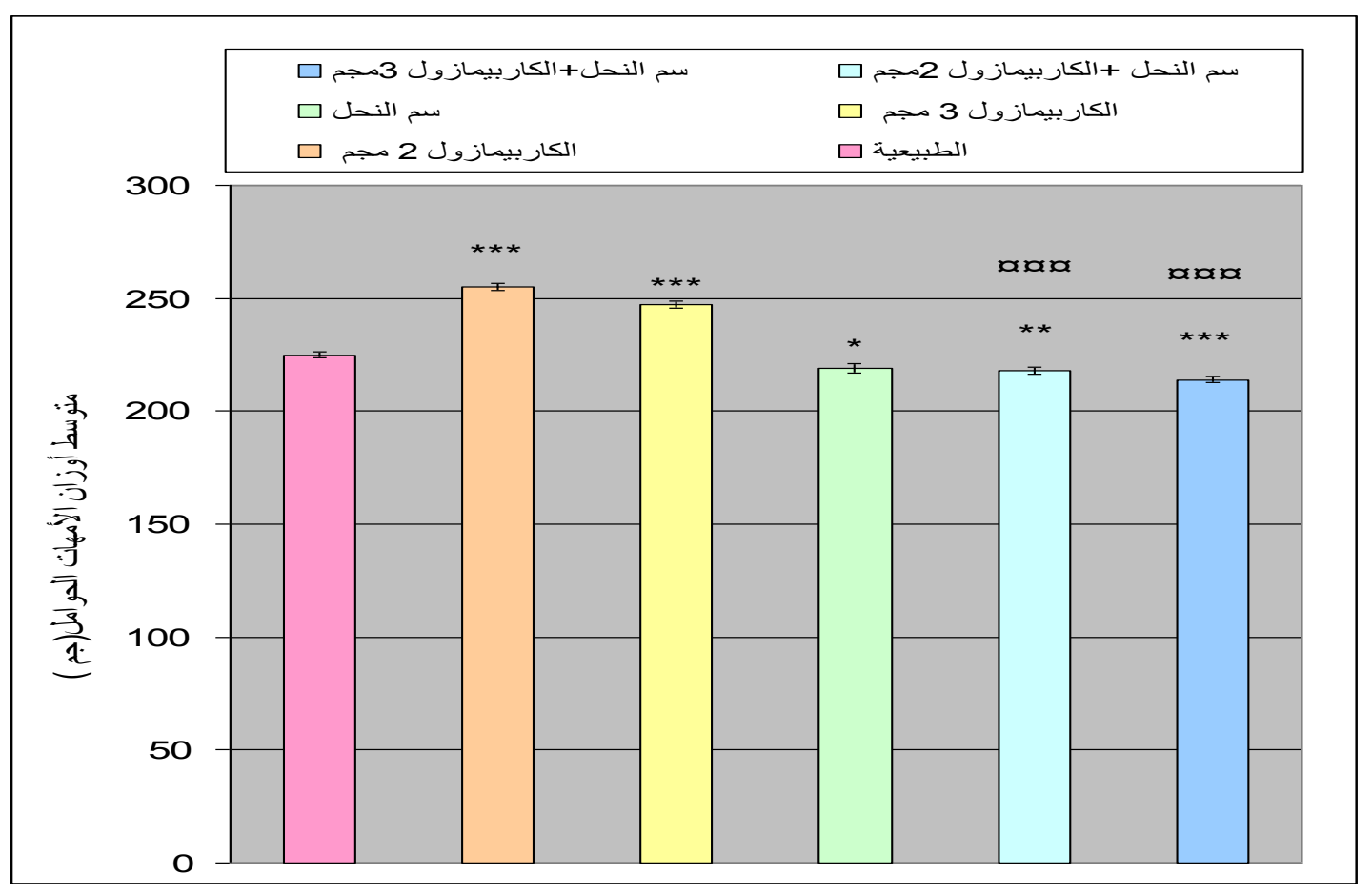

شكل (2): متوسط وزن الرحم بالأجنة

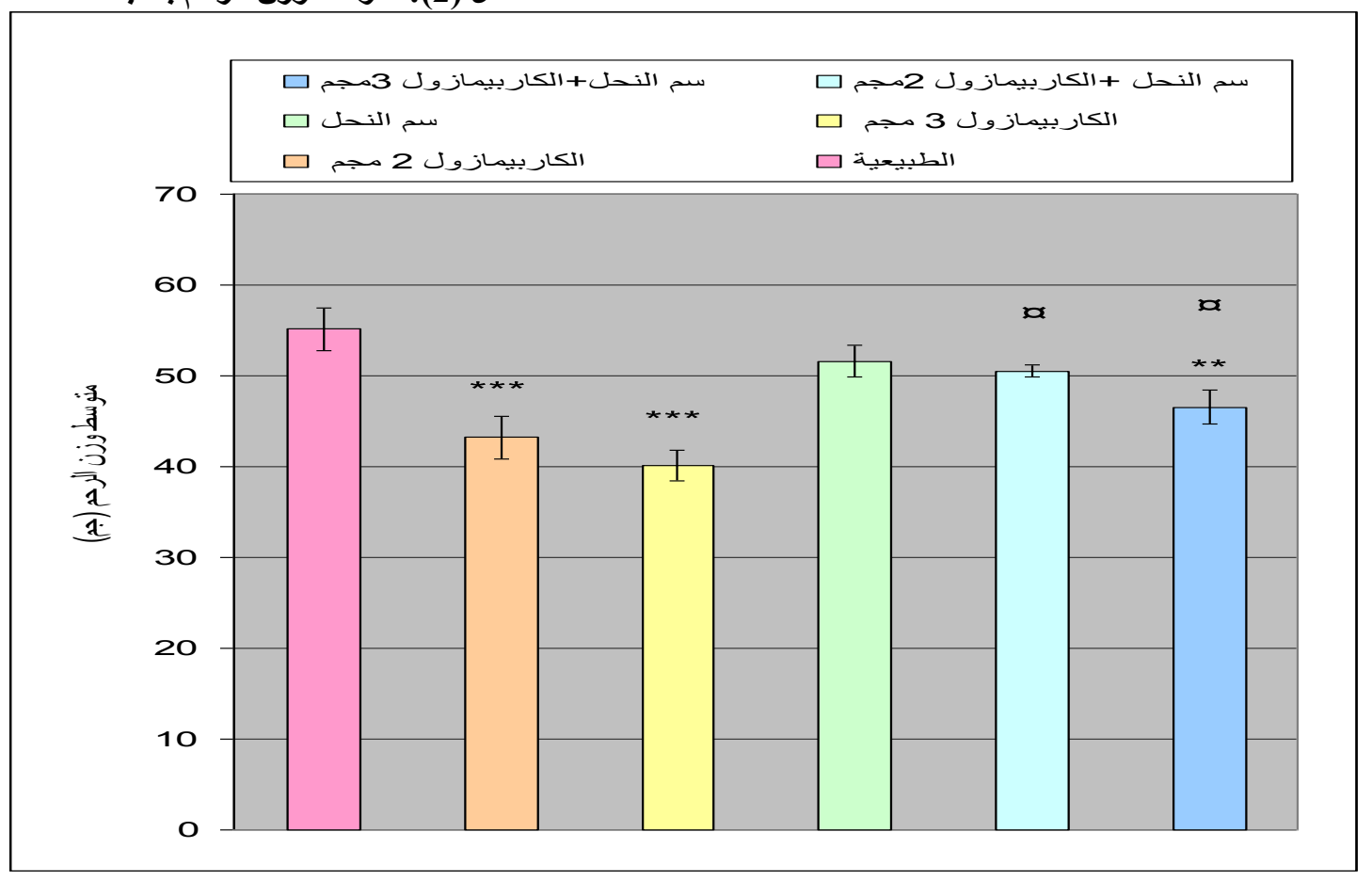


The Role of Bee Venom in Controlling the Morphometric and Morphological Changes...

\section{شكل(3):متوسط أوزان الأجنة}

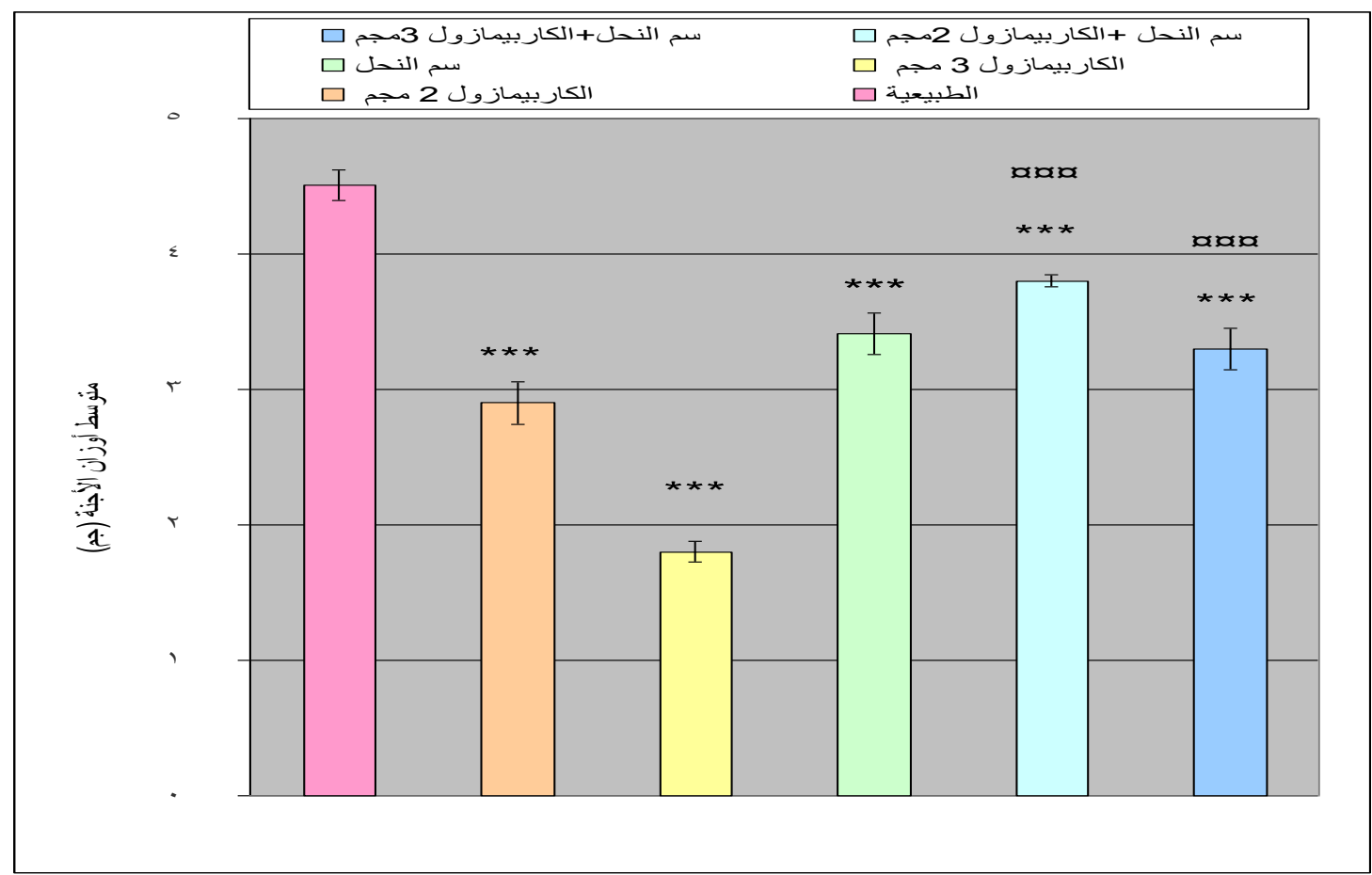

\section{شكل(4):متوسط أطوال الأجنة}

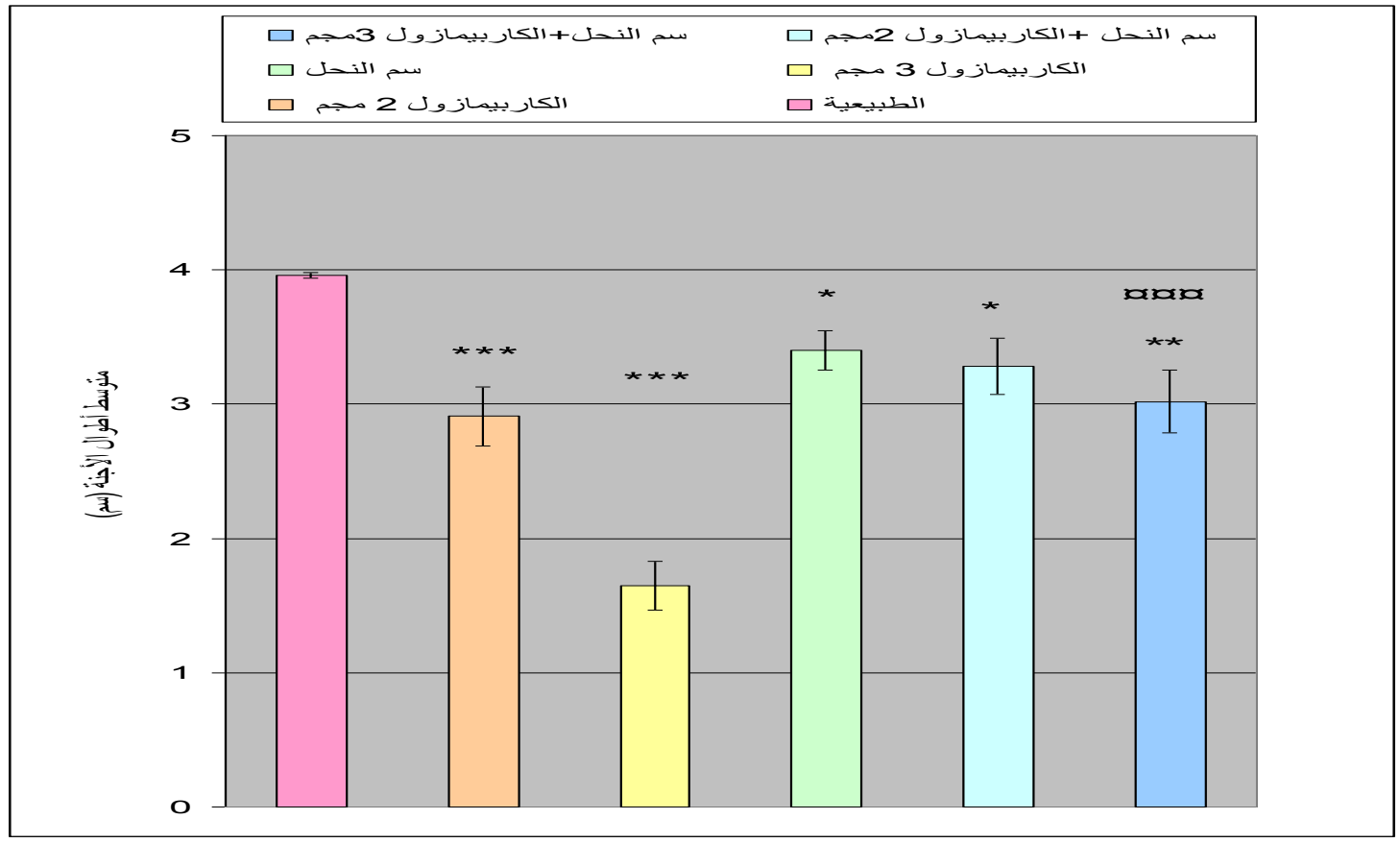




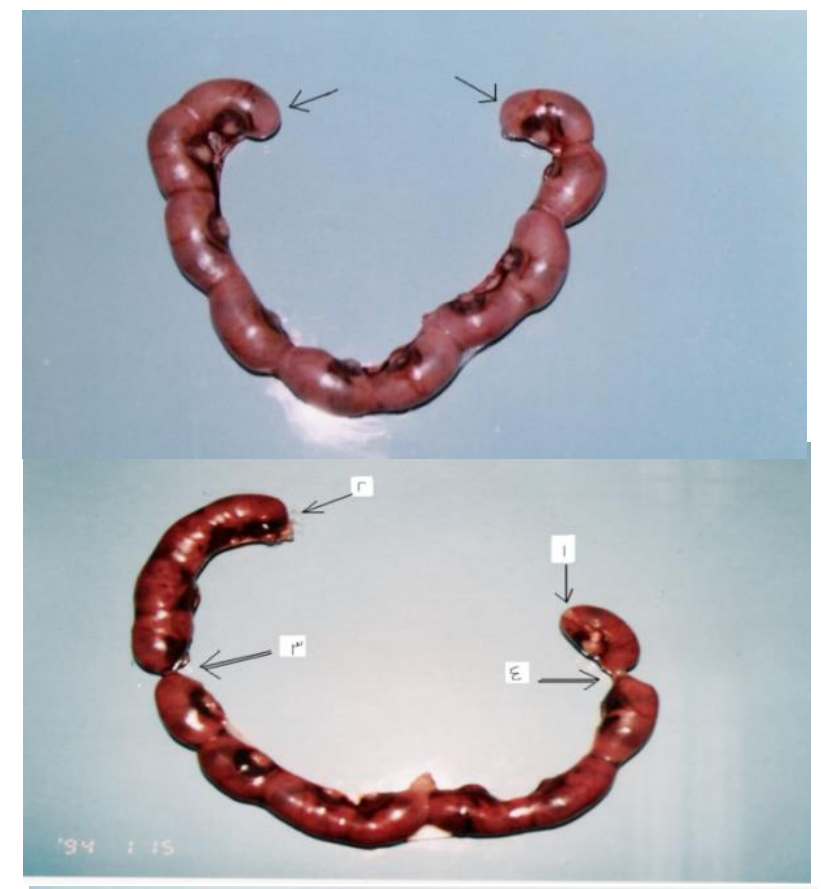

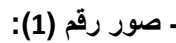

توضح الثكل الخارجي للارحم الطبيعي في الــيوم 19

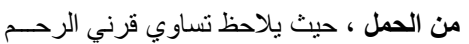

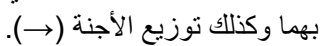
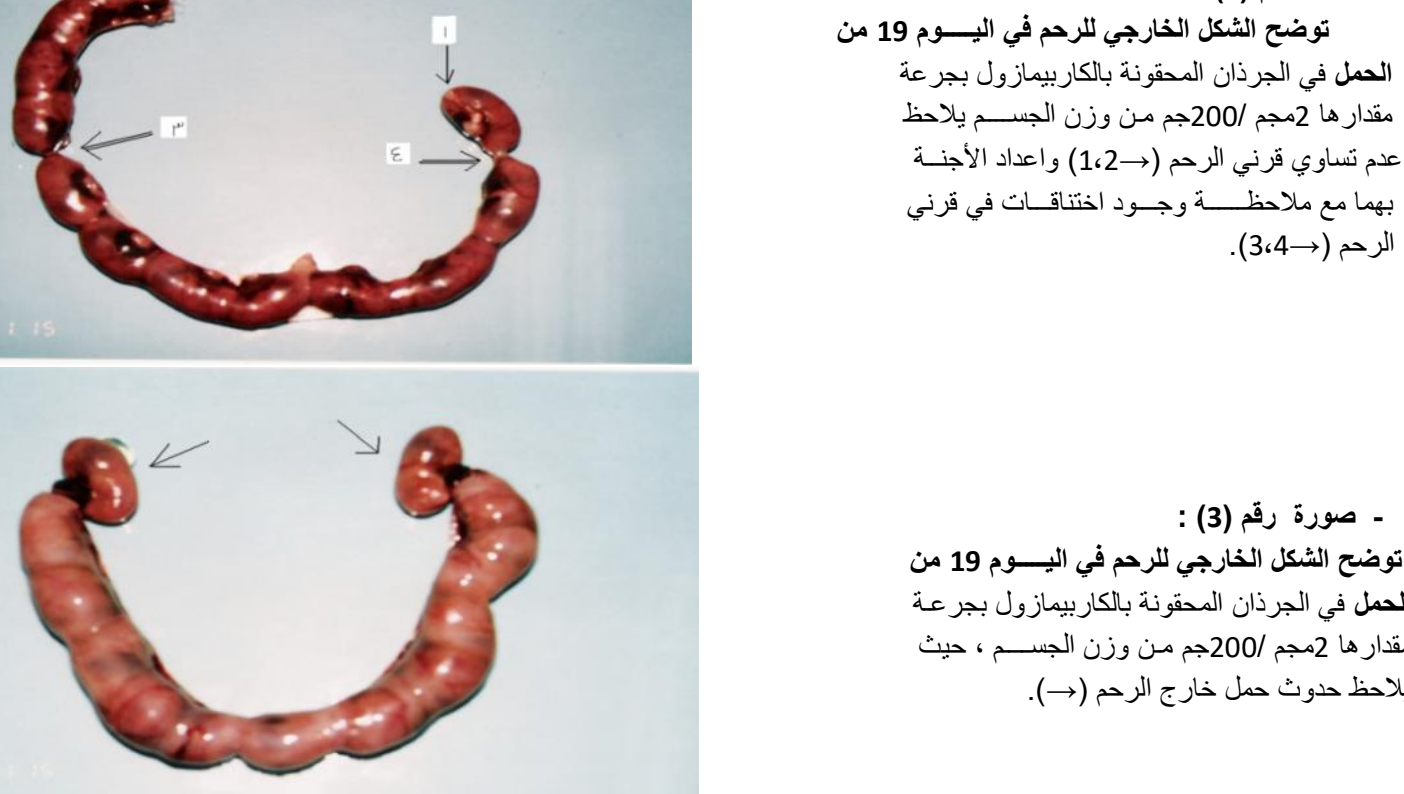

- صورة رقم (3) :

توضح الثكل الخارجي للرحم في اليــــوم 19 من

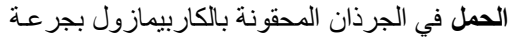

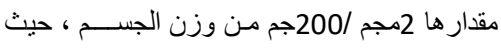

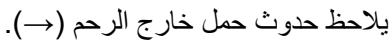

- (4) - صورة رقم (4) : توضح الثكل الخارجي للرحم في اليوم 19 من الحمل

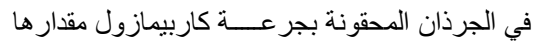

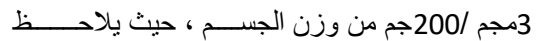

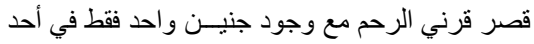

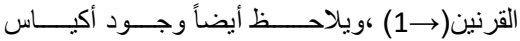

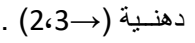


The Role of Bee Venom in Controlling the Morphometric and Morphological Changes...

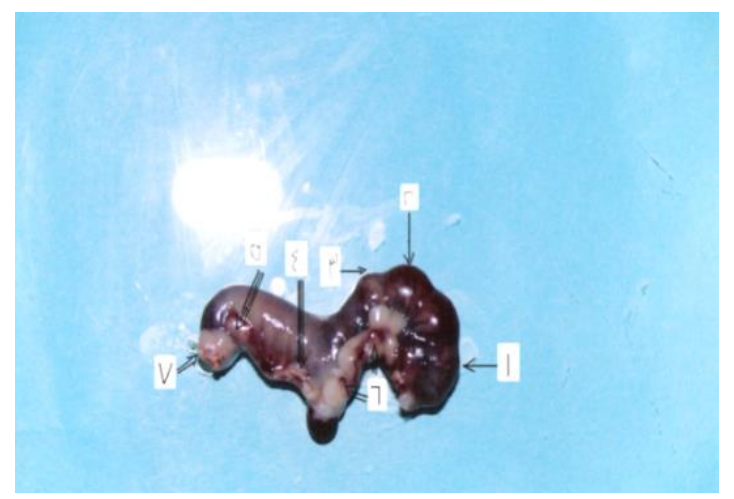

- صورة رقم (5) : توضح الثكل الخارجي للرحم في اليوم 19 من الحمل

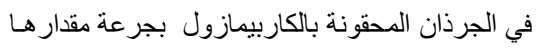

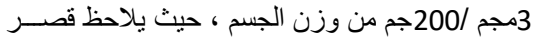
قرني الرحم ( (1) ) ، كما يلاحظ احتو ائهما على بقايـا

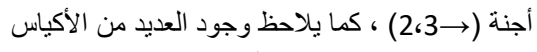

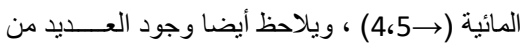

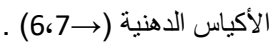

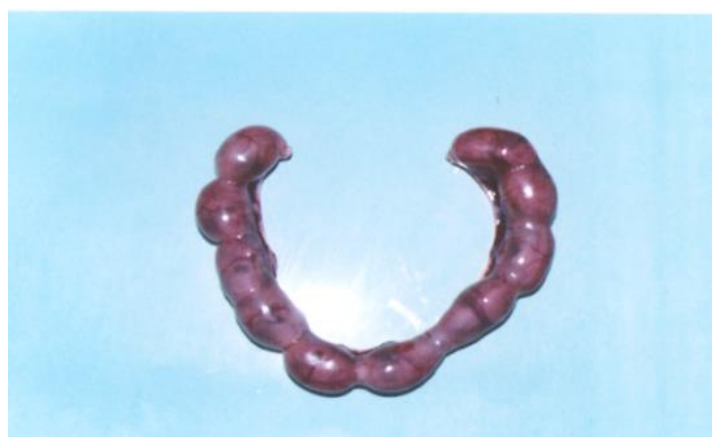

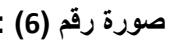
توضح الثكل الخارجي للرحم في اليـوم 19 من

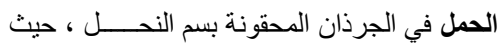
يلاحظ أن الثنكل الخارجي للرحم مشابه للطبيعي .

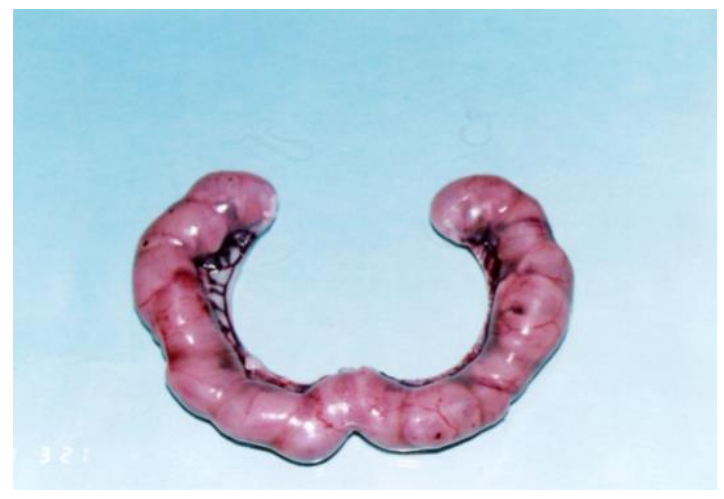

- صورة رقم (7) : توضح الثكل الخارجي للرحم في اليـوم 19 من

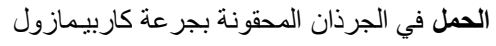

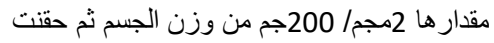

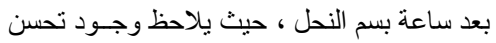

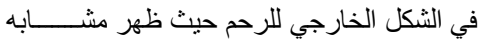
للطبيعي . للإن الخار

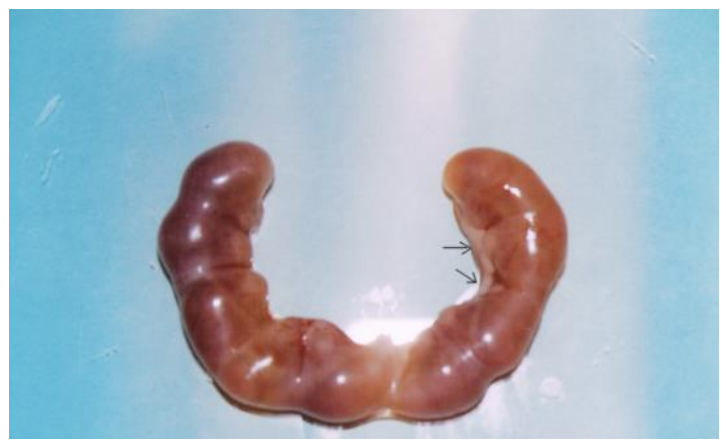

- صورة رقم (8) ) توضح الثكل الخارجي للرحم في اليـــوم 19 من

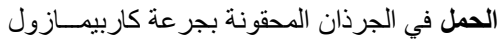

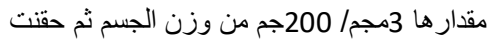

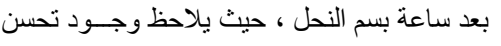

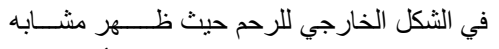

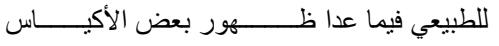

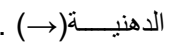




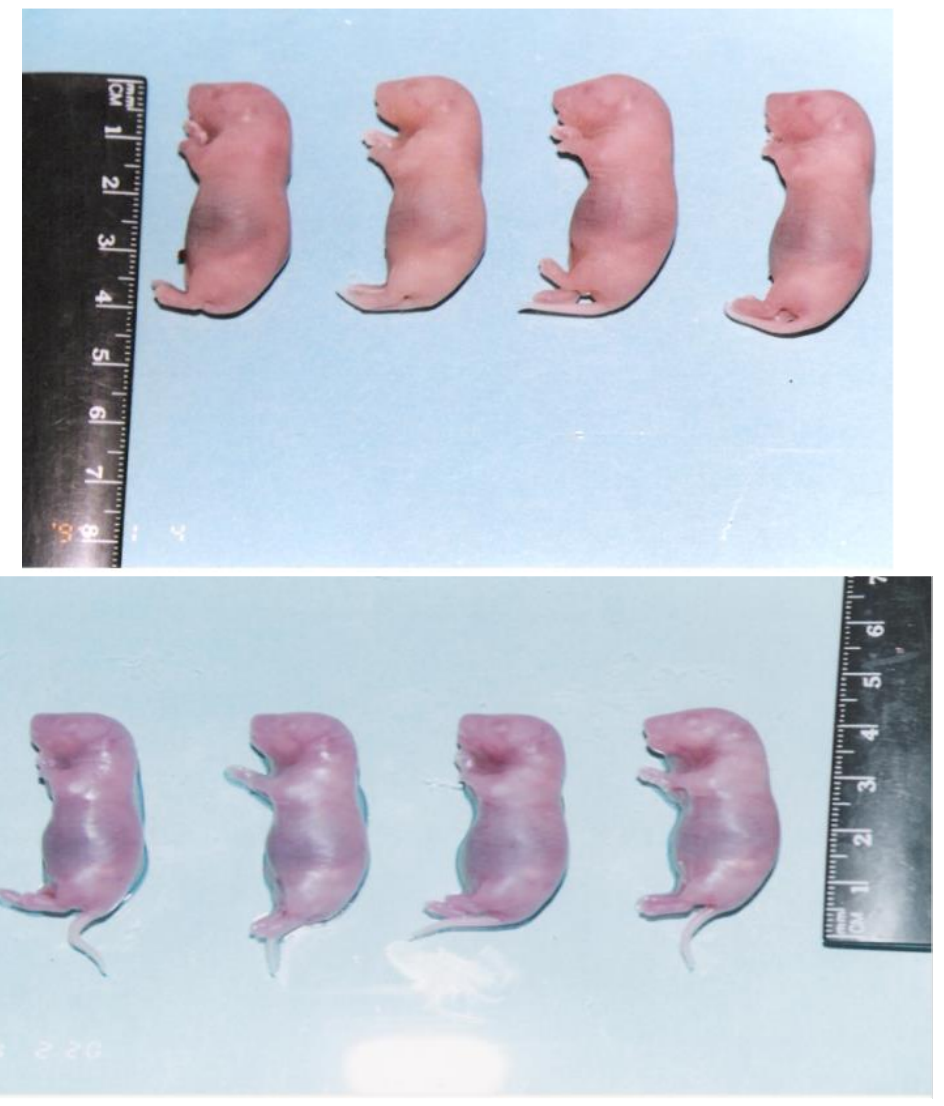

- صورة رقم) -

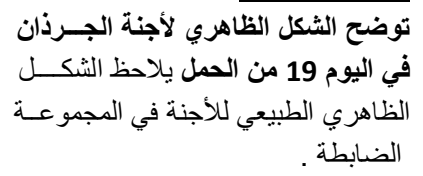

- صورة رقم (10) توضح الشكل الظاهري لأجنة الجرذان في اليوم

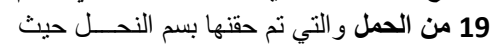

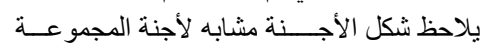

الضابطة .

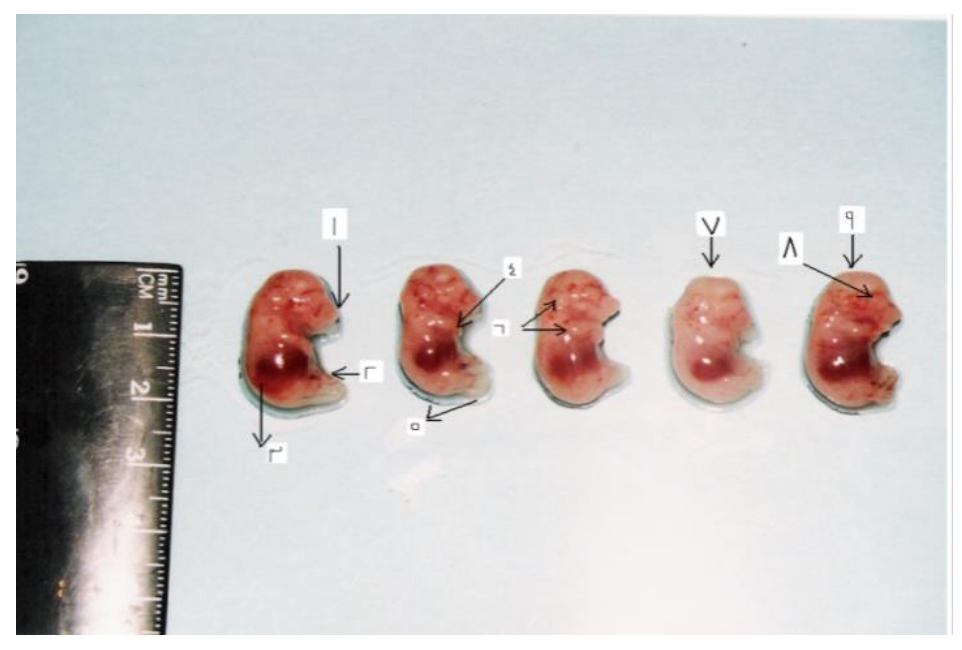

- صوره رقم (11)

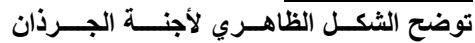

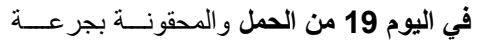

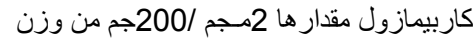

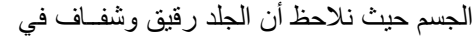

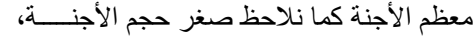

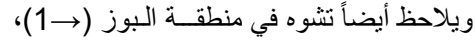

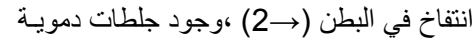

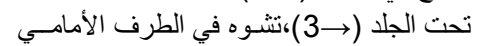

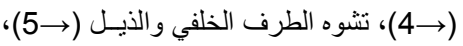

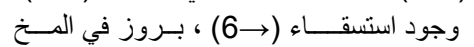

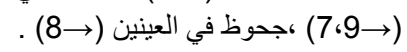


The Role of Bee Venom in Controlling the Morphometric and Morphological Changes...

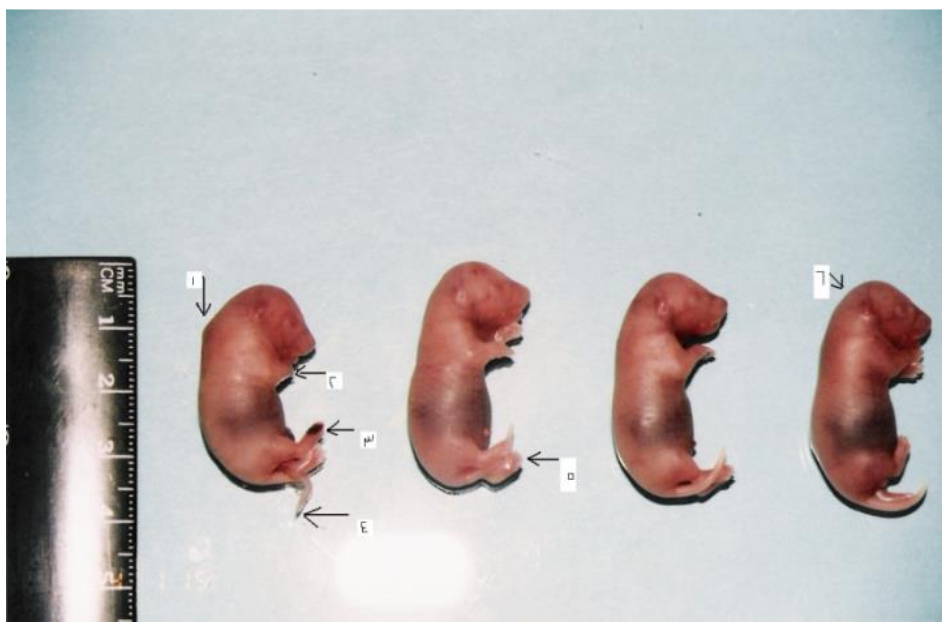

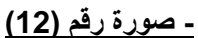

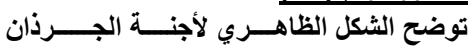

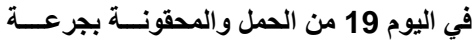

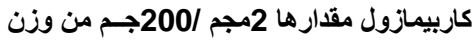
الجسم :نلاحظ وجود انحناء في منطقة العـمود العبمد

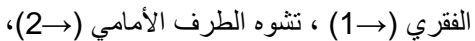

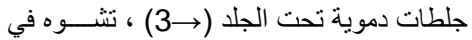

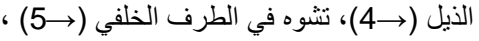
تشوه في الأذن (4)، تشوه

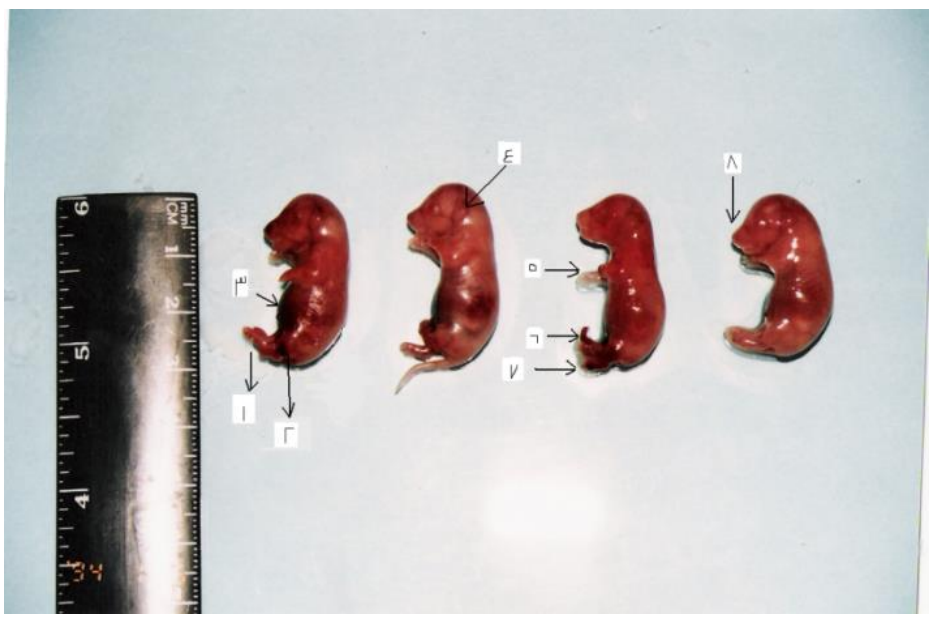

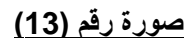

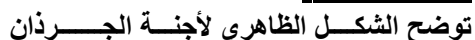

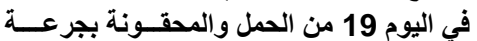

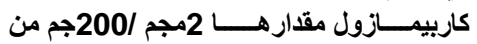
وزن الجسم: يلاحظ أن الجلد رقيق وشفاف

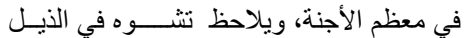

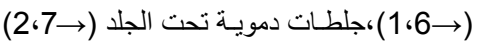

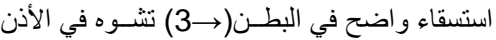

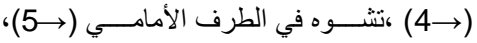

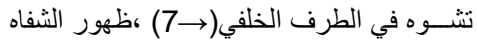
الأرنبية(

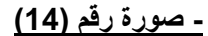
توضح الثكل الظاهري لأجنة الجرذان في اليوم

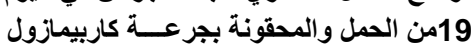

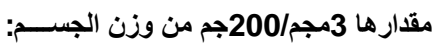
يلاحظ ظهور العديد من بقايا الأجنة . 
Abu Gabal H et al

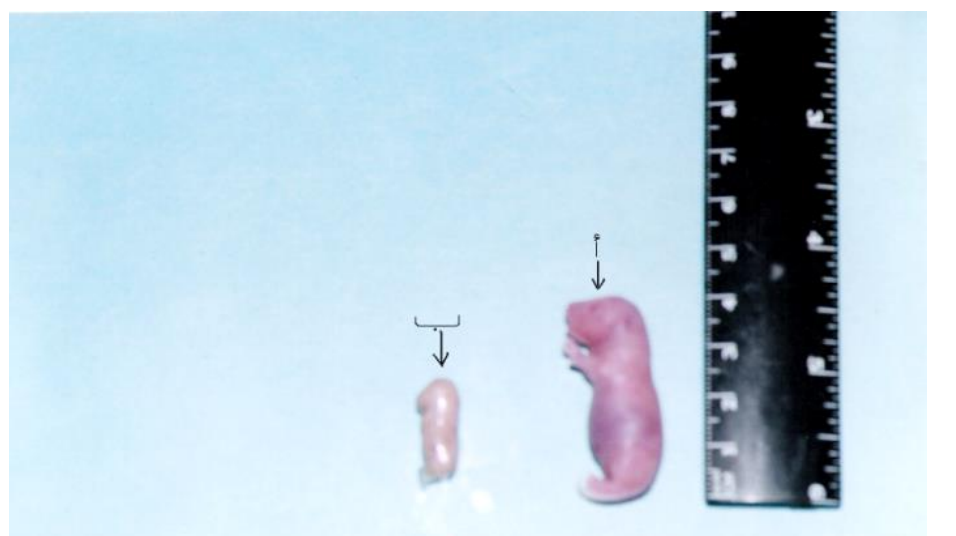

\section{صورة رقم (15) - (15)}

توضح الثكل الظاهري لأجنة الجرذان في اليوم

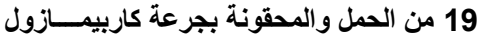
مقارها 3مجم/200جم من وزن الجسم حيث

يلاحظ:

أ-الجلد شفاف ، الثفاه الأرنبية ، انتفـاخ في البطن ،

$$
\text { تنشوه الأطراف ، تثوه الذيل. }
$$

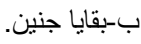

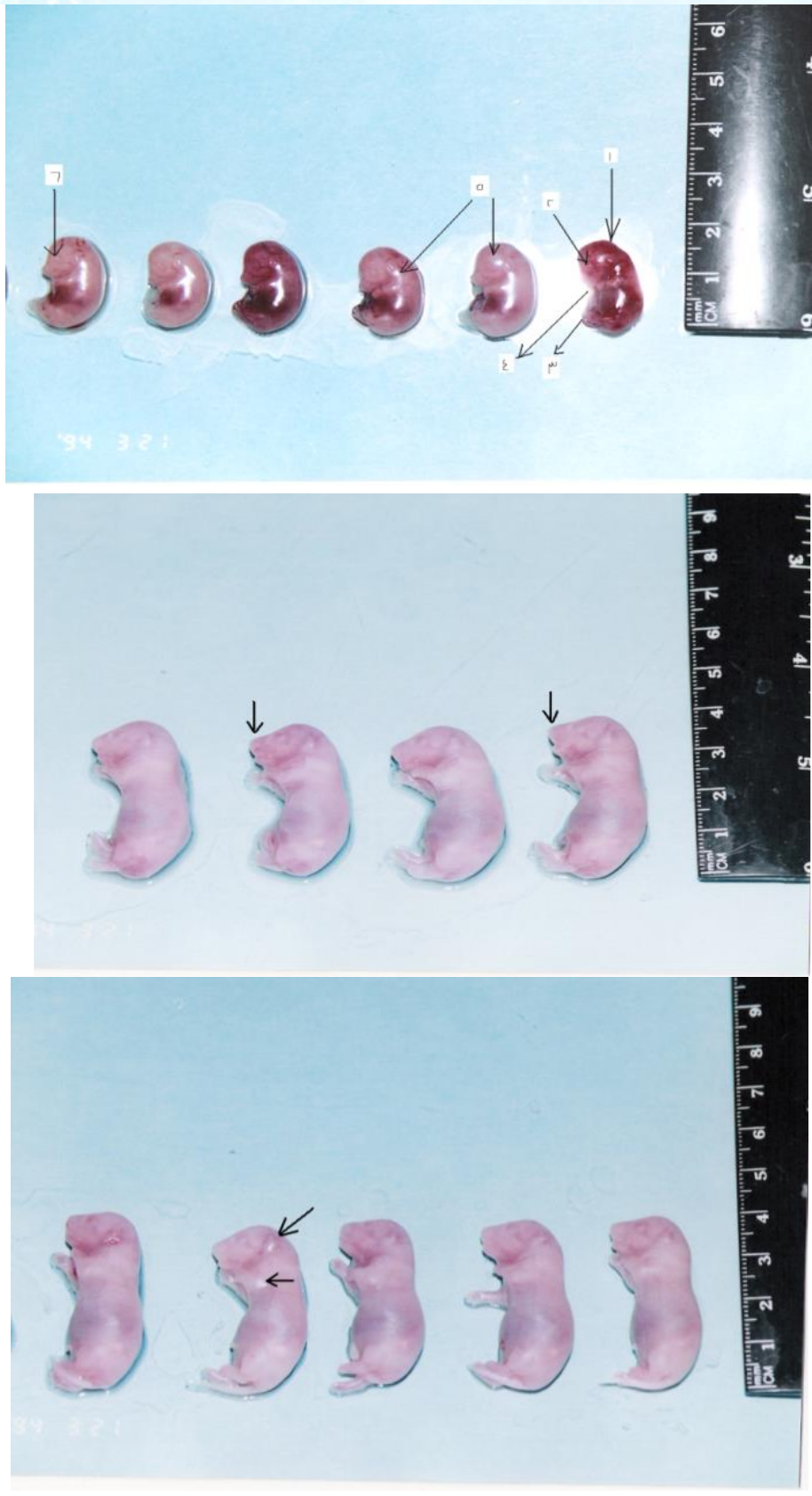

صورة رقم (16)

توضح الثكل الظاهزي لأجنة الجرذان في الــيوم

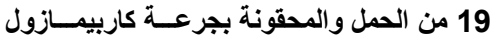

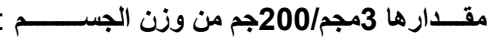
يلاحظ صغر حجم الأجنة ، الجلد شفـــاف

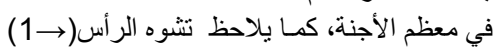

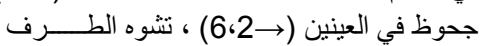

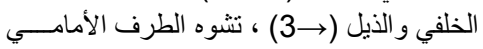

. $(5 \rightarrow)$ ( $)$

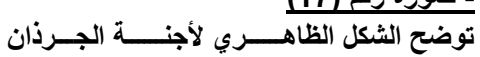

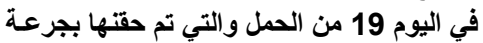

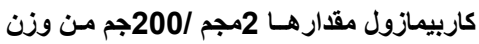

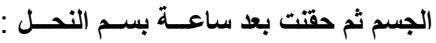
يلاحظ حدوث تحسن في الثكل الظاهري للأجنة باستثناء وجود الثفاه الأرنبية في بعض الأجنـة .

- صورة رقم(18):

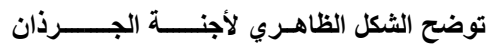

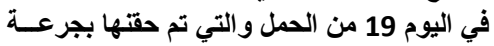

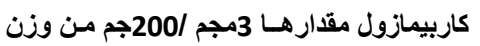

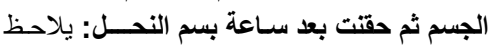

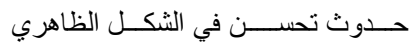
للأجنة باستثناء وجود استسقاء في بعض الأجنة . 